\title{
ONE POSITIVE AND TWO NEGATIVE RESULTS FOR DERIVED CATEGORIES OF ALGEBRAIC STACKS
}

\author{
JACK HALL, AMNON NEEMAN, AND DAVID RYDH
}

\begin{abstract}
Let $X$ be a quasi-compact and quasi-separated scheme. There are two fundamental and pervasive facts about the unbounded derived category of $X:(1) \mathrm{D}_{\mathrm{qc}}(X)$ is compactly generated by perfect complexes and (2) if $X$ is noetherian or has affine diagonal, then the functor $\Psi_{X}: \mathrm{D}(\mathrm{Q} \operatorname{Coh}(X)) \rightarrow$ $\mathrm{D}_{\mathrm{qc}}(X)$ is an equivalence. Our main results are that for algebraic stacks in positive characteristic, the assertions (1) and (2) are typically false.
\end{abstract}

\section{InTRODUCTION}

Fix a field $k$ and an algebraic group $G$ over $k$. Ben-Zvi posed the following question [BZ09]: if $k$ has positive characteristic, then is the unbounded derived category of representations of $G$ compactly generated?

The second author recently answered Ben-Zvi's question negatively in the case of $\mathbb{G}_{a}$ Nee14, Rem. 4.2]. We establish a much stronger version of this result: in the unbounded derived category of representations of $\mathbb{G}_{a}$ in positive characteristic, there are no compact objects besides 0 (Proposition 3.1).

We say that $G$ is poor if $k$ has positive characteristic and $\bar{G}=G \otimes_{k} \bar{k}$ has a subgroup isomorphic to $\mathbb{G}_{a}$, or, equivalently, if $\bar{G}_{\text {red }}^{0}$ is not semi-abelian (Lemma 4.2. Examples of poor groups are $\mathbb{G}_{a}$ and $\mathrm{GL}_{n}$. The results of this article imply that in positive characteristic, the derived category of representations of $G$ is not compactly generated if $G$ is poor. Conversely, when $G$ is not poor, the first and third author showed that its derived category of representations is compactly generated HR15, Thm. A]. Ben-Zvi's question is thus completely resolved.

A somewhat subtle point that we have suppressed so far is that there are two potential ways to look at the unbounded derived category of representations of $G$. First, there is $\mathrm{D}(\operatorname{Rep}(G))$; second, there is $\mathrm{D}_{\mathrm{qc}}(B G)$, the unbounded derived category of lisse-étale $\mathcal{O}_{B G}$-modules with quasi-coherent cohomology. There is a natural functor $\mathrm{D}(\operatorname{Rep}(G)) \rightarrow \mathrm{D}_{\mathrm{qc}}(B G)$ and if $G$ is affine, then this functor induces an equivalence on bounded below derived categories.

In the present article, we will show that in positive characteristic if $G$ is affine and poor, then this functor is not full. We also prove that if $G$ is poor, then neither $\mathrm{D}(\operatorname{Rep}(G))$ nor $\mathrm{D}_{\mathrm{qc}}(B G)$ is compactly generated.

The results above are actually special cases of some general results for unbounded derived categories of quasi-coherent sheaves on algebraic stacks. We say that an algebraic stack is poorly stabilized (see \$4) if it has a point with poor stabilizer group. Our first main result is the following.

Date: September 22, 2017.

2010 Mathematics Subject Classification. Primary 14F05; secondary 13D09, 14A20, $18 \mathrm{G} 10$.

Key words and phrases. Derived categories, algebraic stacks.

The first and second author are supported by the Australian Research Council (ARC), grant numbers FL100100137, DE150101799, and DP150102313.

The third author is supported by the Swedish Research Council (VR), grant numbers 2011-5599 and 2015-05554. 
Theorem 1.1. Let $X$ be an algebraic stack that is quasi-compact, quasi-separated and poorly stabilized.

(1) The triangulated category $\mathrm{D}_{\mathrm{qc}}(X)$ is not compactly generated.

(2) Assume in addition that $X$ has affine diagonal or is noetherian. If $X$ is of global type, then $\mathrm{D}(\mathrm{Q} C \mathrm{Ch}(X))$ is not compactly generated.

An algebraic stack $X$ is of global type if there exists a quasi-compact, étale, representable, and surjective morphism $\left[U / \mathrm{GL}_{n}\right] \rightarrow X$, where $U$ is a quasi-affine scheme Ryd15, §2]. More colloquially, $X$ is of global type if it has affine stabilizers and étale-locally has the resolution property Tot04, Gro17. By Sumihiro's Theoremand its recent generalization due to Brion Sum74, Bri15]-many quotient stacks are of global type [HR17, Prop. 9.1]. So too are stacks admitting good moduli spaces and, more generally, those with linearly reductive stabilizers at closed points AHR15.

We wish to point out that Theorem 1.1 is counter to the prevailing wisdom. Indeed, let $X$ be a quasi-compact and quasi-separated algebraic stack. If $X$ is a scheme, then it is well-known that $\mathrm{D}_{\mathrm{qc}}(X)$ is compactly generated by perfect complexes BB03, Thm. 3.1.1(b)]. More generally, recent work of Krishna Kri09, Lem. 4.8], Ben-Zvi-Francis-Nadler [BZFN10, §3.3], Toën [Toë12, Cor. 5.2], and the first and third authors [HR17, has shown that the unbounded derived category $\mathrm{D}_{\mathrm{qc}}(X)$ is compactly generated by perfect complexes if $X$ is a Deligne-Mumford stack with separated diagonal or is of equicharacteristic zero and of s-global type.

Also recall that if $X$ is a scheme that is either quasi-compact with affine diagonal or noetherian, then the functor $\Psi_{X}: \mathrm{D}(\mathrm{Q} \operatorname{Coh}(X)) \rightarrow \mathrm{D}_{\mathrm{qc}}(X)$ is an equivalence of triangulated categories - see [BN93. Cor. 5.5] for the separated case (the argument adapts trivially to the case of affine diagonal) and [Stacks, Tags 08H1 \& 09TN] in the setting of algebraic spaces. Our second main result is a partial extension of this to algebraic stacks.

Theorem 1.2. Let $X$ be an algebraic stack that is quasi-compact with affine diagonal or noetherian and affine-pointed. If $\mathrm{D}_{\mathrm{qc}}(X)$ is compactly generated, then the functor $\Psi_{X}: \mathrm{D}(\mathrm{Q} \operatorname{Coh}(X)) \rightarrow \mathrm{D}_{\mathrm{qc}}(X)$ is an equivalence of categories.

An algebraic stack $X$ is affine-pointed if every morphism Spec $k \rightarrow X$, where $k$ is a field, is affine. If $X$ has quasi-affine or quasi-finite diagonal, then $X$ is affinepointed [HR14, Lem. 4.5].

In particular, $\Psi_{X}$ is an equivalence for every Deligne-Mumford stack with affine diagonal, every noetherian Deligne-Mumford stack with separated diagonal, and every stack in characteristic zero with affine diagonal that étale-locally has the resolution property HR17. This is a vast extension of work of Lieblich Lie04, Prop. 2.2.4.6] and Krishna [Kri09, Cor. 3.7]. Lieblich gives a sketch of the proof of the equivalence of $\Psi_{X}$ when $X$ is an Artin stack with affine diagonal, the resolution property, and a good moduli space which is a scheme. Krishna treats the special case when $X$ is a Deligne-Mumford stack that is separated, of finite type over a field of characteristic 0 , has the resolution property and whose coarse moduli space is a scheme.

It is natural to ask whether $\Psi_{X}$ is always an equivalence of categories. On the positive side, we prove that the restricted functor $\Psi_{X}^{+}: \mathrm{D}^{+}(\mathrm{Q} \operatorname{Coh}(X)) \rightarrow \mathrm{D}_{\mathrm{qc}}^{+}(X)$ is an equivalence of triangulated categories when either $X$ is quasi-compact with affine diagonal or noetherian and affine-pointed (Theorem C.1, also see [Lur04, Thm. 3.8] and [SGA6, Prop. II.3.5]). On the negative side, we have the following result.

Theorem 1.3. Let $X$ be an algebraic stack that is quasi-compact with affine diagonal or noetherian and affine-pointed. If $X$ is poorly stabilized, then the functor $\Psi_{X}: \mathrm{D}(\mathrm{Q} \operatorname{Coh}(X)) \rightarrow \mathrm{D}_{\mathrm{qc}}(X)$ is not full. 
We were unable to determine whether the functor $\Psi_{X}$ in Theorem 1.3 is faithful or not. For stacks with non-affine stabilizer groups the situation is even worse: if $X=B E$, where $E$ is an elliptic curve over $\mathbb{C}$, then the functor $\Psi_{X}^{b}: \mathrm{D}^{b}(\operatorname{Coh}(X)) \rightarrow$ $\mathrm{D}_{\mathrm{Coh}}^{b}(X)$ is neither essentially surjective nor full.

Note that when $X$ has affine diagonal or is noetherian and affine-pointed, the first claim in Theorem 1.1 is a trivial consequence of Theorems 1.2 and 1.3 .

Left-completeness. In the course of proving Theorem 1.3, we will prove that the triangulated category $\mathrm{D}(\mathrm{Q} \operatorname{Coh}(X))$ is not left-complete whenever $X$ is poorly stabilized with affine diagonal. This generalizes an example of Neeman [Nee11] and amplifies some observations of Drinfeld-Gaitsgory DG13, Rem. 1.2.10].

In Appendix B, we will prove that $\mathrm{D}_{\mathrm{qc}}(X)$ is left-complete for all algebraic stacks $X$. An analogous assertion in the context of derived algebraic geometry has been addressed by Drinfeld-Gaitsgory [DG13, Lem. 1.2.8]. In the Stacks Project Stacks, Tag 08IY] a similar result has been proved, albeit in a different context.

As remarked to us by Bhatt Bha12] and a reviewer, if $X$ is quasi-compact with affine diagonal or noetherian and affine-pointed, then $\mathrm{D}_{\mathrm{qc}}(X)$ can be identified with the left-completion of $\mathrm{D}(\mathrm{Q} \operatorname{Coh}(X))$ (in the sense of [HA, $\S 1.2 .1]$ ) - see Remark C.4.

Well generation. In Appendix $\mathrm{A}$ we show that if $\mathcal{A}$ is a Grothendieck abelian category and $\mathcal{M} \subseteq \mathcal{A}$ is a weak Serre subcategory that is closed under coproducts and is Grothendieck abelian, then $\mathrm{D}_{\mathcal{M}}(\mathcal{A})$ is a well generated triangulated category - a result we expect to be of independent interest. We prove this using the Gabriel-Popescu Theorem.

Since the inclusion $\mathrm{Q} \operatorname{Coh}(X) \subseteq \operatorname{Mod}(X)$ has these properties, this establishes that the triangulated category $\mathrm{D}_{\mathrm{qc}}(X)$ is well generated. This result is applied extensively in the article. It is used in the construction of adjoint functors (e.g., the derived quasi-coherator) and infinite products.

As remarked by a reviewer, the well generation of $\mathrm{D}_{\mathcal{M}}(\mathcal{A})$ also follows from some general results in the theory of presentable $\infty$-categories (Remark A.4). We also wish to point out that while [KS06, Prop. 14.2.4] is quite general, it does not apply in our situation. Indeed, they require that the embedding $\mathcal{M} \subseteq \mathcal{A}$ is closed under $\mathcal{A}$-subquotients (i.e., $\mathcal{M}$ is a Serre subcategory of $\mathcal{A}$ ), which is not the case for $\mathrm{Q} \operatorname{Coh}(X) \subseteq \operatorname{Mod}(X)$.

Acknowledgements. We wish to thank Bhargav Bhatt and Jacob Lurie for answering some questions and Peter O'Sullivan for providing a reference. We are also indebted to the reviewer for a number of useful comments and suggestions.

\section{Preliminaries}

Let $\phi: X \rightarrow Y$ be a quasi-compact and quasi-separated morphism of algebraic stacks. Then the restriction of the functor $\left(\phi_{\text {lis-ét }}\right)_{*}: \operatorname{Mod}(X) \rightarrow \operatorname{Mod}(Y)$ to $\mathrm{QCoh}(X)$ factors through $\mathrm{QCoh}(Y)$ Ols07, Lem. 6.5(i)], giving rise to a functor $\left(\phi_{\mathrm{QCoh}}\right)_{*}: \mathrm{Q} \operatorname{Coh}(X) \rightarrow \mathrm{Q} \operatorname{Coh}(Y)$. Since the categories $\operatorname{Mod}(X)$ and $\mathrm{Q} \operatorname{Coh}(X)$ are Grothendieck abelian Stacks, Tag 0781], the unbounded derived functors of $\left(\phi_{\text {lis-ét }}\right)_{*}$ and $\left(\phi_{\mathrm{QCoh}}\right)_{*}$ exist Stacks, Tags 079P \& 070K], and we denote these as $\mathrm{R}\left(\phi_{\text {lis-ét }}\right)_{*}$ and $\mathrm{R}\left(\phi_{\mathrm{Q} \text { Coh }}\right)_{*}$, respectively. By [Ols07, Lem. 6.20], the restriction of $\mathrm{R}\left(\phi_{\text {lis-ét }}\right)_{*}$ to $\mathrm{D}_{\mathrm{qc}}^{+}(X)$ factors uniquely through $\mathrm{D}_{\mathrm{qc}}^{+}(Y)$. If, in addition, $\phi$ is concentrated (e.g., representable), then the restriction of $\mathrm{R}\left(\phi_{\text {lis-ét }}\right)_{*}$ to $\mathrm{D}_{\mathrm{qc}}(X)$ factors through $\mathrm{D}_{\mathrm{qc}}(Y)$ (see [Hal14, Lem. 2.1] for the representable case and HR17, Thm. 2.6(ii)] in general). 
For an algebraic stack $W$ let $\Psi_{W}: \mathrm{D}(\mathrm{Q} \operatorname{Coh}(W)) \rightarrow \mathrm{D}_{\mathrm{qc}}(W)$ denote the natural functor. The universal properties of right-derived functors provide a diagram:

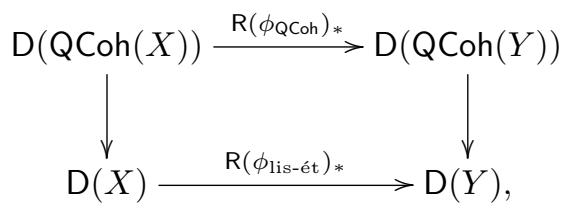

together with a natural transformation of functors:

$$
\epsilon_{\phi}: \Psi_{Y} \circ \mathrm{R}\left(\phi_{\mathrm{QCoh}}\right)_{*} \Rightarrow \mathrm{R}\left(\phi_{\text {lis-ét }}\right)_{*} \circ \Psi_{X} .
$$

The following result, for schemes, is well-known [TT90, B.8]; for algebraic spaces, see [Stacks, Tags 09TH \& 08GX].

Proposition 2.1. Let $\phi: X \rightarrow Y$ be a morphism of algebraic stacks. Suppose that both $X$ and $Y$ are quasi-compact with affine diagonal or noetherian and affinepointed. If $M \in \mathrm{D}^{+}(\mathrm{Q} \operatorname{Coh}(X))$, then the morphism induced by (2.1):

$$
\epsilon_{\phi}(M): \Psi_{Y} \circ \mathrm{R}\left(\phi_{\mathrm{QCoh}}\right)_{*}(M) \rightarrow \mathrm{R}\left(\phi_{\text {lis-ét }}\right)_{*} \circ \Psi_{X}(M)
$$

is an isomorphism. In particular, since $\Psi_{Y}^{+}: \mathrm{D}^{+}(\mathrm{Q} \operatorname{Coh}(Y)) \rightarrow \mathrm{D}_{\mathrm{qc}}^{+}(Y)$ is an equivalence (Theorem C.1), it follows that there is a natural isomorphism for each $M \in \mathrm{D}^{+}(\mathrm{Q} \operatorname{Coh}(X)):$

$$
\mathrm{R}\left(\phi_{\mathrm{QCoh}}\right)_{*}(M) \rightarrow\left(\Psi_{Y}^{+}\right)^{-1} \circ \mathrm{R}\left(\phi_{\text {lis-ét }}\right)_{*} \circ \Psi_{X}^{+}(M) .
$$

Proof. The functors $\left(\phi_{\mathrm{QC} \text { Coh }}\right)_{*}$ and $\left(\phi_{\text {lis-ét }}\right)_{*}$ are left-exact, thus the functors $\mathrm{R}\left(\phi_{\mathrm{Q} C o h}\right)_{*}$ and $\mathrm{R}\left(\phi_{\text {lis-ét }}\right)_{*}$ are bounded below. Since $M$ is assumed to belong to the bounded below derived category, standard "way-out" arguments show that it is sufficient to prove the result in the case when $M \simeq N[0]$, where $N \in \mathrm{Q} \operatorname{Coh}(X)$. The isomorphism, in this case, reduces to proving that if $N \in \mathrm{Q} \operatorname{Coh}(X)$, then the natural morphism $\mathrm{R}^{i}\left(\phi_{\mathrm{QCoh}}\right)_{*} N \rightarrow \mathrm{R}^{i}\left(\phi_{\text {lis-ét }}\right)_{*} N$ is an isomorphism for all integers $i \geq 0$, where $\mathrm{R}^{i}\left(\phi_{\mathrm{QCoh}}\right)_{*}\left(\right.$ resp. $\left.\mathrm{R}^{i}\left(\phi_{\text {lis-ét }}\right)_{*}\right)$ denotes the $i$ th right-derived functor of $\left(\phi_{\mathrm{QCoh}}\right)_{*}$ (resp. $\left.\left(\phi_{\text {lis-ét }}\right)_{*}\right)$. A standard $\delta$-functor argument shows that it is sufficient to prove that $\mathrm{R}^{i}\left(\phi_{\text {lis-ét }}\right)_{*} I=0$ for every $i>0$ and injective $I$ of $\mathrm{QCoh}(X)$. But $X$ and $Y$ are assumed to be either quasi-compact with affine diagonal or noetherian and affine-pointed, so this vanishing claim follows from Lemma C.32).

We briefly recall the following definitions from [HR17, §2]. Let $\phi: X \rightarrow Y$ be a quasi-compact and quasi-separated morphism of algebraic stacks. Then $\phi$ has finite cohomological dimension if there exists an integer $n>0$ such that $\mathrm{R}^{m}\left(\phi_{\text {lis-ét }}\right)_{*} M=$ 0 for all $m \geq n$ and $M \in \mathrm{Q} \operatorname{Coh}(X)$. If for every morphism of algebraic stacks $Z \rightarrow Y$, where $Z$ is quasi-compact and quasi-separated, the morphism $X \times_{Y} Z \rightarrow Z$ has finite cohomological dimension, then we say that $\phi$ is concentrated.

If $Y$ is quasi-compact with quasi-affine diagonal, then finite cohomological dimension is equivalent to concentrated [HR17, Lem. 2.5(v)]. Also, if $\phi$ is representable, then it is concentrated HR17, Lem. 2.5(iii)].

Concentrated morphisms are the natural ones to consider for unbounded derived categories of quasi-coherent sheaves. Indeed, if $\phi$ is concentrated, then $\mathrm{R}\left(\phi_{\text {lis-ét }}\right)_{*}$ sends $\mathrm{D}_{\mathrm{qc}}(X)$ to $\mathrm{D}_{\mathrm{qc}}(Y)$, is compatible with flat base change and preserves small coproducts [HR17, Thm 2.6]. In the next corollary, we see that this is also often the case for $\mathrm{R}\left(\phi_{\mathrm{QCoh}}\right)_{*}$.

Corollary 2.2. Let $\phi: X \rightarrow Y$ be a concentrated morphism of algebraic stacks. If $X$ and $Y$ are quasi-compact with affine diagonal or noetherian and affine-pointed, 
then there exists an integer $r \geq 0$ such that for all $M \in \mathrm{D}(\mathrm{Q} \operatorname{Coh}(X))$ and integers $n$ the natural map:

$$
\tau^{\geq n} \mathrm{R}\left(\phi_{\mathrm{QCoh}}\right)_{*} M \rightarrow \tau^{\geq n} \mathrm{R}\left(\phi_{\mathrm{QCoh}}\right)_{*} \tau^{\geq n-r} M
$$

is a quasi-isomorphism. It follows that

(1) $\mathrm{R}\left(\phi_{\mathrm{QCoh}}\right)_{*}$ preserves small coproducts;

(2) for all $M \in \mathrm{D}(\mathrm{Q} C \mathrm{Co}(X))$ the natural morphism induced by (2.1):

$$
\epsilon_{\phi}(M): \Psi_{Y} \circ \mathrm{R}\left(\phi_{\mathrm{QCoh}}\right)_{*} M \rightarrow \mathrm{R}\left(\phi_{\text {lis-ét }}\right)_{*} \circ \Psi_{X}(M)
$$

is an isomorphism;

(3) the formation of $\mathrm{R}\left(\phi_{\mathrm{QCoh}}\right)_{*}$ is compatible with flat base change on $Y$; and

(4) if a left adjoint $\mathrm{L} \phi_{\mathrm{Q} C o h}^{*}$ to $\mathrm{R}\left(\phi_{\mathrm{QCoh}}\right)_{*}$ exists (see Lemma 4.3. e.g., $\phi$ is flat), then $\mathrm{L} \phi_{\mathrm{Q}}^{*} \mathrm{coh}$ sends compacts to compacts.

Proof. Since $\phi$ is a concentrated morphism and $Y$ is quasi-compact and quasiseparated, there exists an integer $r \geq 0$ such that if $N \in \mathrm{Q} \operatorname{Coh}(X)$, then $\mathrm{R}^{i}\left(\phi_{\text {lis-ét }}\right)_{*} N=$ 0 for all $i>r$. By Proposition 2.1 it follows that $\mathrm{R}^{i}\left(\phi_{\mathrm{QCoh}}\right)_{*} N=0$ for all $i>r$ too. The claim now follows from [Stacks, Tag 07K7]. The claims (1)-(3) are all simple consequences of the main claim, Proposition 2.1 and HR17, Thm 2.6]. Finally, (4) follows from (1) and [HR17, Ex. 3.8].

Corollary 2.3. Let $X$ be an algebraic stack that is quasi-compact with affine diagonal or noetherian and affine-pointed. If $C$ is a compact object of either $\mathrm{D}(\mathrm{QCoh}(X))$ or $\mathrm{D}_{\mathrm{qc}}(X)$, then $C$ is perfect. Moreover if $X$ is noetherian, then $C$ is quasiisomorphic to a bounded complex of coherent sheaves on $X$.

Proof. Let $C$ be a compact object of $\mathrm{D}_{\mathrm{qc}}(X)$. By [HR17, Lem. 4.4(i)], $C$ is a perfect complex and in particular belongs to $\mathrm{D}_{\mathrm{qc}}^{b}(X) \subseteq \mathrm{D}_{\mathrm{qc}}^{+}(X)$. By Theorem C.1 it follows that $C \simeq \Psi_{X}(\tilde{C})$ for some $\tilde{C} \in \mathrm{D}^{b}(\mathrm{Q} \operatorname{Coh}(X))$. If $X$ is noetherian, $\tilde{C}$ even belongs to $\mathrm{D}_{\operatorname{Coh}(X)}^{b}(\mathrm{Q} \operatorname{Coh}(X))$. Combining [LMB, Prop. 15.4] with [SGA6, II.2.2], we deduce that $C$ belongs to the image of $\mathrm{D}(\operatorname{Coh}(X)) \rightarrow \mathrm{D}_{\mathrm{qc}}(X)$.

Now let $C$ be a compact object of $\mathrm{D}(\mathrm{Q} \operatorname{Coh}(X))$. Let $p: U \rightarrow X$ be a smooth surjection from an affine scheme $U$. By Corollary 2.2 4 , $\mathrm{L} p_{\mathrm{Q} C o h}^{*} C \in \mathrm{D}(\mathrm{Q} C o h(U))$ is compact. Since $U=\operatorname{Spec} A$ is affine, it follows that $\mathrm{Q} \operatorname{Coh}(U) \cong \operatorname{Mod}(A)$ and so $\mathrm{L} p_{\mathrm{Q} \text { Coh }}^{*} C$ is a perfect complex [Stacks, Tag 07LT]. If $X$ is noetherian, then $C \in$ $\mathrm{D}_{\mathrm{Coh}(X)}^{b}(\mathrm{Q} \operatorname{Coh}(X))$. Arguing as before, we deduce that $C$ belongs to the image of $\mathrm{D}(\operatorname{Coh}(X)) \rightarrow \mathrm{D}(\mathrm{Q} \operatorname{Coh}(X))$.

In the following Lemma we will give a sufficient condition for compactness of a perfect object in $\mathrm{D}(\mathrm{Q} \operatorname{Coh}(X))$. We do not know if this condition is necessary. The analogous condition in $\mathrm{D}_{\mathrm{qc}}(X)$ is necessary [HR17, Lem. 4.5].

Lemma 2.4. Let $X$ be an algebraic stack that is quasi-compact with affine diagonal or noetherian and affine-pointed. Let $P \in \mathrm{D}(\mathrm{Q} \operatorname{Coh}(X))$ be a perfect complex. Consider the following conditions

(1) $P$ is a compact object of $\mathrm{D}(\mathrm{Q} \operatorname{Coh}(X))$.

(2) There exists an integer $r \geq 0$ such that $\operatorname{Hom}_{\mathcal{O}_{X}}(P, N[i])=0$ for all $N \in$ $\mathrm{Q} \operatorname{Coh}(X)$ and $i>r$.

(3) There exists an integer $r \geq 0$ such that the natural map

$$
\tau^{\geq j} \operatorname{RHom}_{\mathrm{D}(\mathrm{Q} \operatorname{Coh}(X))}(P, M) \rightarrow \tau^{\geq j} \operatorname{RHom}_{\mathrm{D}(\mathrm{Q} \operatorname{Coh}(X))}\left(P, \tau^{\geq j-r} M\right)
$$

is a quasi-isomorphism for all $M \in \mathrm{D}(\mathrm{Q} \operatorname{Coh}(X))$ and integers $j$.

Then (2) and (3) are equivalent and imply (1). 
Proof. Condition (2) is a special case of (3): let $M=N[i]$ and $j=0$.

Conversely, assume that condition (2) holds and let $M \in \mathrm{D}(\mathrm{Q} \operatorname{Coh}(X))$. Since the category $\mathrm{Q} C o h(X)$ is Grothendieck abelian, there is a quasi-isomorphism $M \rightarrow I^{\bullet}$ in $\mathrm{D}(\mathrm{Q} C o h(X))$, where $I^{\bullet}$ is K-injective and $I^{j}$ is injective for every integer $j$ Ser03.

Let $p \geq r+1$ be an integer with the property that $P \in \mathrm{D}^{\geq-p+1}(\mathrm{Q} \operatorname{Coh}(X))$. Then the natural morphism of chain complexes:

$$
\tau^{\geq j} \operatorname{Hom}_{\mathrm{K}(\mathrm{Q} \operatorname{Coh}(X))}\left(P, I^{\bullet}\right) \rightarrow \tau^{\geq j} \operatorname{Hom}_{\mathrm{K}(\mathrm{Q} \operatorname{Coh}(X))}\left(P, \sigma^{\geq j-p} I^{\bullet}\right),
$$

where $\sigma$ is the brutal truncation, is a quasi-isomorphism. For every integer $j$ there is also a morphism $s_{j}: \sigma^{\geq j} I^{\bullet} \rightarrow \tau^{\geq j} I^{\bullet}$. If $C_{j}^{\bullet}$ is the mapping cone of $s_{j}$, then $C_{j}^{\bullet} \simeq d\left(I^{j-1}\right)[-(j-1)]$. Thus, by condition [2], it follows that for every integer $j$

$$
\tau^{\geq j+r} \operatorname{RHom}_{\mathrm{D}(\mathrm{Q} \operatorname{Coh}(X))}\left(P, C_{j}^{\bullet}\right) \simeq 0 .
$$

Since there is also a distinguished triangle in $\mathrm{D}(\mathrm{Q} \operatorname{Coh}(X))$ for every integer $j$ :

$$
\operatorname{RHom}\left(P, \sigma^{\geq j-p} I^{\bullet}\right) \longrightarrow \operatorname{RHom}\left(P, \tau^{\geq j-p} I^{\bullet}\right) \longrightarrow \operatorname{RHom}\left(P, C_{j-p}^{\bullet}\right),
$$

it follows that for every integer $j$ there is a quasi-isomorphism:

$$
\tau^{\geq j} \operatorname{RHom}\left(P, \sigma^{\geq j-p} I^{\bullet}\right) \simeq \tau^{\geq j} \operatorname{RHom}\left(P, \tau^{\geq j-p} M\right) .
$$

For every integer $j$, we also have a distinguished triangle

$$
H^{j-r-1}(M)[-(j-r-1)] \longrightarrow \tau^{\geq j-r-1} M \longrightarrow \tau^{\geq j-r} M .
$$

As before, it follows that $\tau^{\geq j} \operatorname{RHom}\left(P, \tau^{\geq j-r-1} M\right) \simeq \tau^{\geq j} \operatorname{RHom}\left(P, \tau^{\geq j-r} M\right)$ and thus by induction a quasi-isomorphism:

$$
\tau^{\geq j} \operatorname{RHom}\left(P, \tau^{\geq j-p} M\right) \simeq \tau^{\geq j} \operatorname{RHom}\left(P, \tau^{\geq j-r} M\right) .
$$

Combining the quasi-isomorphisms (2.2)-2.4 gives (3).

For (3) implies (1): this follows from Theorem C.1 and [HR17, Lem. 1.2(iii)].

We now relate compact generation in $\mathrm{D}(\mathrm{Q} \operatorname{Coh}(X))$ with compact generation in $\mathrm{D}_{\mathrm{qc}}(X)$.

Lemma 2.5. Let $X$ be an algebraic stack that is quasi-compact with affine diagonal or noetherian and affine-pointed.

(1) If $P \in \mathrm{D}(\mathrm{Q} \operatorname{Coh}(X))$ is a perfect complex such that $\Psi(P)$ is compact in $\mathrm{D}_{\mathrm{qc}}(X)$, then $P$ is compact in $\mathrm{D}(\mathrm{Q} \operatorname{Coh}(X))$.

(2) If $X$ has finite cohomological dimension, then every perfect complex is compact in both $\mathrm{D}(\mathrm{Q} C o h(X))$ and $\mathrm{D}_{\mathrm{qc}}(X)$.

(3) If a set of objects $\left\{P_{i}\right\}$ of $\mathrm{D}(\mathrm{QCoh}(X))$ has the property that $\left\{\Psi\left(P_{i}\right)\right\}$ compactly generates $\mathrm{D}_{\mathrm{qc}}(X)$, then $\left\{P_{i}\right\}$ compactly generates $\mathrm{D}(\mathrm{Q} \operatorname{Coh}(X))$.

Proof. For (1), by [HR17, Lem. 4.5], since $\Psi(P)$ is compact, there exists an integer $r$ such that if $i>r$ and $N \in \mathrm{Q} \operatorname{Coh}(X)$, then $\operatorname{Hom}_{\mathcal{O}_{X}}(\Psi(P), N[i])=0$. The functor $\Psi^{+}$is an equivalence (Theorem C.1), so $\operatorname{Hom}_{\mathcal{O}_{X}}(P, N[i])=0$ for all $i>r$ and $N \in \mathrm{Q} C o h(X)$. It follows that $P$ is compact by Lemma 2.4 .

Statement (2) is a direct consequence of (1) and [HR17, Lem. 4.4(iii)].

For (3), let $M \in \mathrm{D}(\mathrm{Q} \operatorname{Coh}(X))$. If $P$ is perfect and $\Psi(P)$ is compact, then $\operatorname{RHom}(P, M)=\operatorname{RHom}(\Psi(P), \Psi(M))$. Indeed, there exists an integer $r$ such that for all integers $j$

$$
\begin{aligned}
\tau^{\geq j} \operatorname{RHom}(P, M) & \simeq \tau^{\geq j} \operatorname{RHom}\left(P, \tau^{\geq j-r} M\right) \\
& \simeq \tau^{\geq j} \operatorname{RHom}\left(\Psi(P), \tau^{\geq j-r} \Psi(M)\right) \simeq \tau^{\geq j} \operatorname{RHom}(\Psi(P), \Psi(M)),
\end{aligned}
$$

by Lemma 2.4 and HR17, Lem. 4.5] since $\Psi^{+}$is an equivalence of triangulated categories (Theorem C.1) and $\Psi$ is t-exact. Thus, if $\operatorname{Hom}_{\mathrm{D}(\mathrm{Q} \operatorname{Coh}(X))}\left(P_{i}[l], M\right)=0$ 
for all $i$ and integers $l$, then $\operatorname{Hom}_{\mathcal{O}_{X}}\left(\Psi\left(P_{i}\right)[l], \Psi(M)\right)=0$ for all $i$ and $l$. It follows that $\Psi(M)=0$ and, since $\Psi$ is conservative, that $M=0$.

The following lemma, while technical, gives an explicit description of an adjunction that is useful in the article.

Lemma 2.6. Let $X$ be an algebraic stack and let $M \in \mathrm{D}(\mathrm{Q} \operatorname{Coh}(X))$.

(1) The functor $\Psi_{X}: \mathrm{D}(\mathrm{Q} C o h(X)) \rightarrow \mathrm{D}_{\mathrm{qc}}(X)$ admits a right adjoint $\Phi_{X}: \mathrm{D}_{\mathrm{qc}}(X) \rightarrow$ $\mathrm{D}(\mathrm{Q} \operatorname{Coh}(X))$.

(2) If $X$ is quasi-compact with affine diagonal or noetherian and affine-pointed, then there exists a compatible quasi-isomorphism:

$$
\Phi_{X} \Psi_{X}(M) \simeq \underset{n}{\operatorname{holim}} \tau^{\geq-n} M .
$$

Proof. We suppress the subscript $X$ from $\Psi$ and $\Phi$ throughout. Since $\Psi$ preserves small coproducts and $\mathrm{D}(\mathrm{Q} \operatorname{Coh}(X))$ is well generated [Nee01a, Thm. 0.2], $\Psi$ admits a right adjoint $\Phi: \mathrm{D}_{\mathrm{qc}}(X) \rightarrow \mathrm{D}(\mathrm{Q} \operatorname{Coh}(X))$ Nee01b, Prop. 1.20]. This proves (1).

To prove (2), by left-completeness of $\mathrm{D}_{\mathrm{qc}}(X)$ (Theorem B.1),

$$
\Phi \Psi(M) \rightarrow \Phi\left(\operatorname{holim}_{n} \tau^{\geq-n} \Psi(M)\right)
$$

is a quasi-isomorphism. Since $\Phi$ is a right adjoint, it preserves homotopy limits. Also, $\Psi$ is t-exact. Hence, there is a quasi-isomorphism

$$
\Phi\left(\underset{n}{\operatorname{holim}} \tau^{\geq-n} \Psi(M)\right) \simeq \underset{n}{\operatorname{holim}} \Phi \Psi\left(\tau^{\geq-n} M\right) .
$$

By Theorem C.1. however, $\tau^{\geq-n} M \simeq \Phi \Psi\left(\tau^{\geq-n} M\right)$. This proves the claim.

Remark 2.7. From Lemma 2.6 2) it is immediate that when $X$ is quasi-compact with affine diagonal or is noetherian and affine-pointed, the left-completeness of $\mathrm{D}(\mathrm{Q} \operatorname{Coh}(X))$ is equivalent to $\Psi_{X}$ being fully faithful.

We now prove Theorem 1.2 using an argument similar to [BIK11, Lem. 4.5].

Proof of Theorem 1.2. By Lemma 2.5 (3), both $\mathrm{D}(\mathrm{Q} C o h(X))$ and $\mathrm{D}_{\mathrm{qc}}(X)$ are compactly generated and $\Psi$ takes a set of compact generators to a set of compact generators. In particular, the right adjoint $\Phi: \mathrm{D}_{\mathrm{qc}}(X) \rightarrow \mathrm{D}(\mathrm{Q} \operatorname{Coh}(X))$ of $\Psi$ preserves small coproducts Nee96, Thm. 5.1].

Consider the unit $\left.\eta_{M}: M \rightarrow \Phi \Psi(M)\right)$ and the counit $\epsilon_{M}: \Psi \Phi(M) \rightarrow M$ of the adjunction. Since $\Psi^{+}$is an equivalence, we have that $\eta_{P}$ and $\epsilon_{P}$ are isomorphisms for every compact object $P$. Since $\Psi$ and $\Phi$ preserve small coproducts and $\mathrm{D}_{\mathrm{qc}}(X)$ and $\mathrm{D}(\mathrm{Q} C o h(X))$ are compactly generated, it follows that $\eta$ and $\epsilon$ are equivalences. We conclude that $\Psi$ is an equivalence.

\section{The CASE OF $B_{k} \mathbb{G}_{a}$ IN POSITIVE CHARACTERISTIC}

Throughout this section we let $k$ denote a field of characteristic $p>0$. Let $B_{k} \mathbb{G}_{a}$ be the algebraic stack classifying $\mathbb{G}_{a}$-torsors over $k$. We remind ourselves that the category of quasi-coherent sheaves on $B_{k} \mathbb{G}_{a}$ is the category of $\mathbb{G}_{a}$-modules, which is equivalent to the category of locally small modules over a certain ring $R$. In fact $R$ is the ring

$$
R=\frac{k\left[x_{1}, x_{2}, x_{3}, \ldots\right]}{\left(x_{1}^{p}, x_{2}^{p}, x_{3}^{p}, \ldots\right)}
$$

and a module is locally small if every element is annihilated by all but finitely many $x_{i}$ DG70, II.2.2.6(b)]. Let us write $\mathrm{D}\left(R^{\mathrm{ls}}\right)$ for the derived category of the category of locally small $R$-modules, and observe that $\mathrm{D}\left(R^{\mathrm{ls}}\right) \cong \mathrm{D}\left(\mathrm{Q} \operatorname{Coh}\left(B_{k} \mathbb{G}_{a}\right)\right)$.

Proposition 3.1. The only compact objects, in either $\mathrm{D}\left(\mathrm{Q} \operatorname{Coh}\left(B_{k} \mathbb{G}_{a}\right)\right)$ or $\mathrm{D}_{\mathrm{qc}}\left(B_{k} \mathbb{G}_{a}\right)$, are the zero objects. 
Proof. The algebraic stack $B_{k} \mathbb{G}_{a}$ is noetherian with affine diagonal and so, by Corollary 2.3, every compact object is the image of a bounded complex of coherent sheaves. Let $C$ be a compact object; we need to show that $C$ vanishes.

Our compact object $C$ is the image of a finite complex of finitely generated modules in $\mathrm{D}\left(R^{\mathrm{ls}}\right)$. In particular, there exists an integer $n>1$ such that $x_{i}$ annihilates $C$ for all $i \geq n$. Let us put this slightly differently: consider the ring homomorphisms $S \stackrel{\alpha}{\rightarrow} T \stackrel{\beta}{\rightarrow} R \stackrel{\gamma}{\rightarrow} T$ where

$$
S=k\left[x_{n}\right] /\left(x_{n}^{p}\right), \quad T=\frac{k\left[x_{1}, x_{2}, \ldots, x_{n-1}, x_{n}\right]}{\left(x_{1}^{p}, x_{2}^{p}, \ldots, x_{n-1}^{p}, x_{n}^{p}\right)}
$$

where the maps $S \stackrel{\alpha}{\rightarrow} T \stackrel{\beta}{\rightarrow} R$ are the natural inclusions, and where $\gamma: R \rightarrow T$ is defined by

$$
\gamma\left(x_{i}\right)= \begin{cases}x_{i} & \text { if } i \leq n \\ 0 & \text { if } i>n\end{cases}
$$

Note that $\gamma \beta=$ id. Restriction of scalars gives induced maps of derived categories, which we write as $\mathrm{D}(T) \stackrel{\gamma_{*}}{\longrightarrow} \mathrm{D}\left(R^{\mathrm{ls}}\right) \stackrel{\beta_{*}}{\longrightarrow} \mathrm{D}(T) \stackrel{\alpha_{*}}{\longrightarrow} \mathrm{D}(S)$, and $\beta_{*} \gamma_{*}=$ id. Our complex $C$, which is a bounded complex annihilated by $x_{i}$ for all $i \geq n$, is of the form $\gamma_{*} B$ where $B \in \mathrm{D}^{b}(T)$ is a bounded complex of finite $T$-modules. And the fact that $x_{n}$ annihilates $C$ translates to saying that $\alpha_{*} B$ is a complex of modules annihilated by $x_{n}$, that is a complex of $k$-vector spaces. We wish to show that $C=0$ or, equivalently, that $\alpha_{*} B$ is acyclic. We will show that if $C$ is non-zero, then this gives rise to a contradiction.

Thus, assume that the cohomology of $\alpha_{*} B$ is non-trivial: in $\mathrm{D}(S)$ the complex $\alpha_{*} B$ is isomorphic to a non-zero sum of suspensions $k[\ell]$ of $k$. Then there are infinitely many integers $m$ and non-zero maps in $\mathrm{D}(S)$ of the form $\alpha_{*} B \rightarrow k[m]$. Indeed, $\operatorname{Ext}_{S}^{m}(k, k) \neq 0$ for all $m \geq 0$. But $\alpha_{*}$ has a right adjoint $\alpha^{\times}=\operatorname{RHom}_{S}(T,-)$, and we deduce infinitely many non-zero maps in $\mathrm{D}(T)$ of the form $B \rightarrow \alpha^{\times} k[m]=$ $\operatorname{Hom}_{S}(T, k)[m]$ (this is because $T$ is a finite flat $S$-algebra). Since $\mathrm{D}(T)$ is leftcomplete, these combine to a map in $\mathrm{D}(T)$

$$
\Psi: B \rightarrow \prod_{m} \operatorname{Hom}_{S}(T, k)[m] \cong \coprod_{m} \operatorname{Hom}_{S}(T, k)[m]
$$

for which the composites

$$
B \stackrel{\Psi}{\rightarrow} \coprod_{m} \operatorname{Hom}_{S}(T, k)[m] \stackrel{\pi_{m}}{\rightarrow} \operatorname{Hom}_{S}(T, k)[m]
$$

are non-zero. Applying $\gamma_{*}$, which preserves coproducts, we deduce maps

$$
\gamma_{*} B \stackrel{\gamma_{*} \Psi}{\longrightarrow} \coprod_{m} \gamma_{*} \operatorname{Hom}_{S}(T, k)[m] \stackrel{\gamma_{*} \pi_{m}}{\longrightarrow} \gamma_{*} \operatorname{Hom}_{S}(T, k)[m]
$$

whose composites cannot vanish in $\mathrm{D}\left(R^{\mathrm{ls}}\right)$, since $\beta_{*}$ takes them to non-zero maps. The equivalence $\mathrm{D}\left(R^{\mathrm{ls}}\right) \cong \mathrm{D}\left(\mathrm{Q} \operatorname{Coh}\left(B_{k} \mathbb{G}_{a}\right)\right)$ gives us that the composites in $\mathrm{D}\left(\mathrm{Q} \operatorname{Coh}\left(B_{k} \mathbb{G}_{a}\right)\right)$ do not vanish. Furthermore, the composites lie in $\mathrm{D}^{+}\left(\mathrm{Q} \operatorname{Coh}\left(B_{k} \mathbb{G}_{a}\right)\right) \subseteq \mathrm{D}\left(\mathrm{QCoh}\left(B_{k} \mathbb{G}_{a}\right)\right)$, and on $\mathrm{D}^{+}\left(\mathrm{Q} \operatorname{Coh}\left(B_{k} \mathbb{G}_{a}\right)\right)$ the map to $\mathrm{D}_{\mathrm{qc}}\left(B_{k} \mathbb{G}_{a}\right)$ is fully faithful [Lur04, Thm. 3.8]. Hence the images of the composites are non-zero in $\mathrm{D}_{\mathrm{qc}}\left(B_{k} \mathbb{G}_{a}\right)$ as well. But this contradicts the compactness of $C=\gamma_{*} B$.

\section{THE GENERAL CASE}

In this section we extend the results of the previous section and show that the presence of $\mathbb{G}_{a}$ in the stabilizer groups of an algebraic stack $X$ is an obstruction to compact generation in positive characteristic. The existence of finite unipotent subgroups such as $\mathbb{Z} / p \mathbb{Z}$ and $\boldsymbol{\alpha}_{p}$ is an obstruction to the compactness of the structure sheaf $\mathcal{O}_{X}$ but does not rule out compact generation [HR15]. The only 
connected groups in characteristic $p$ without unipotent subgroups are the groups of multiplicative type. The following well-known lemma characterizes the groups without $\mathbb{G}_{a}$ 's.

Lemma 4.1. Let $G$ be a group scheme of finite type over an algebraically closed field $k$. Then the following are equivalent:

(1) $G_{\mathrm{red}}^{0}$ is semiabelian, that is, a torus or the extension of an abelian variety by a torus;

(2) there is no subgroup $\mathbb{G}_{a} \hookrightarrow G$.

Proof. By Chevalley's Theorem Con02, Thm. 1.1] there is an extension $1 \rightarrow H \rightarrow$ $G_{\text {red }}^{0} \rightarrow A \rightarrow 1$ where $H$ is smooth, affine and connected and $A$ is an abelian variety. A subgroup $\mathbb{G}_{a} \hookrightarrow G$ would have to be contained in $H$ which implies that $H$ is not a torus. Conversely, recall that $H(k)$ is generated by its semi-simple and unipotent elements by the Jordan Decomposition Theorem [Bor91, Thm. 4.4]. If $H$ is not a torus, then there exist non-trivial unipotent elements in $H(k)$. But any non-trivial unipotent element of $H(k)$ lies in a subgroup $\mathbb{G}_{a} \hookrightarrow G$. The result follows.

If $k$ is of positive characteristic, then we say that $G$ is poor if $G_{\mathrm{red}}^{0}$ is not semiabelian. We say that an algebraic stack $X$ is poorly stabilized if there exists a geometric point $x$ of $X$ whose residue field $\kappa(x)$ is of characteristic $p>0$ and stabilizer group scheme $G_{x}$ is poor. In particular, the algebraic stacks $B_{k} \mathbb{G}_{a}$ and $B_{k} \mathrm{GL}_{n}$ for $n>1$ are poorly stabilized in positive characteristic. The following characterization of poorly stabilized algebraic stacks will be useful.

Lemma 4.2. Let $X$ be a quasi-separated algebraic stack.

(1) The stack $X$ is poorly stabilized if and only if there exists a field $k$ of characteristic $p>0$ and a representable morphism $\phi: B_{k} \mathbb{G}_{a} \rightarrow X$.

(2) If $X$ has affine stabilizers, then every representable morphism $\phi: B_{k} \mathbb{G}_{a} \rightarrow$ $X$ is quasi-affine.

(3) Let $X^{\prime} \rightarrow X$ be a quasi-finite, representable and surjective morphism of algebraic stacks. If $X$ is poorly stabilized, then so too is $X^{\prime}$.

Proof. We first prove (1). Let $k$ be an algebraically closed field and let $x:$ Spec $k \rightarrow$ $X$ be a geometric point with stabilizer group scheme $G$. This induces a representable morphism $B G \rightarrow X$. If $X$ is poorly stabilized, then there exists a point $x$ such that $G_{\text {red }}^{0}$ is not semiabelian. By the previous lemma, there is a subgroup $\mathbb{G}_{a} \hookrightarrow G$ and hence a representable morphism $B \mathbb{G}_{a} \rightarrow B G$.

Conversely, given a representable morphism $\phi: B_{k} \mathbb{G}_{a} \rightarrow X$, there is an induced representable morphism $\psi: B_{k} \mathbb{G}_{a} \rightarrow B_{k} G$. The morphism $\psi$ is induced by some subgroup $\mathbb{G}_{a} \hookrightarrow G$ (unique up to conjugation) so $X$ is poorly stabilized.

We now treat (2). The structure morphism $\iota_{x}: \mathcal{G}_{x} \hookrightarrow X$ of the residual gerbe $\mathcal{G}_{x}$ at $x$ is quasi-affine Ryd11, Thm. B.2] and $\phi=\iota_{x} \circ \rho \circ \psi$ where $\rho: B_{k} G \rightarrow \mathcal{G}_{x}$ is affine. If $X$ has affine stabilizers, then $G$ is affine and it follows that the quotient $G / \mathbb{G}_{a}$ is quasi-affine since $\mathbb{G}_{a}$ is unipotent [Ros61, Thm. 3]. We conclude that the morphism $\psi: B_{k} \mathbb{G}_{a} \rightarrow B_{k} G$, as well as $\phi$, is quasi-affine.

For (3), using (1) we obtain a morphism $\phi: B_{k} \mathbb{G}_{a} \rightarrow X$, where $k$ is a field of characteristic $p>0$. Let $W=X^{\prime} \times{ }_{X} B_{k} \mathbb{G}_{a}$. The resulting projection $W \rightarrow B_{k} \mathbb{G}_{a}$ is quasi-finite, representable and surjective. In particular, $W \neq \emptyset$. Let $w: \operatorname{Spec} l \rightarrow W$ be a point, where $l$ is an algebraically closed field of characteristic $p>0$; then the stabilizer $G_{w}$ of $w$ is a subgroup scheme of $\left(\mathbb{G}_{a}\right)_{l}$ of finite index. But $\left(\mathbb{G}_{a}\right)_{l}$ is connected, so $G_{w}=\left(\mathbb{G}_{a}\right)_{l}$. It follows from (1) that $X^{\prime}$ is also poorly stabilized.

We now prove Theorem 1.1 . 
Proof of Theorem 1.1. By Lemma 4.2 there exists a field of characteristic $p>0$ and a quasi-compact, quasi-separated and representable morphism $\phi: B_{k} \mathbb{G}_{a} \rightarrow X$.

If $\mathrm{D}_{\mathrm{qc}}(X)$ is compactly generated, then there is a compact object $M \in \mathrm{D}_{\mathrm{qc}}(X)$ and a non-zero map $M \rightarrow \mathrm{R}\left(\phi_{\text {lis-ét }}\right)_{*} \mathcal{O}_{B_{k} \mathbb{G}_{a}}$. Indeed, $\mathrm{R}\left(\phi_{\text {lis-ét }}\right)_{*} \mathcal{O}_{B_{k} \mathbb{G}_{a}} \in \mathrm{D}_{\mathrm{qc}}(X)$ and is non-zero. By adjunction, there is a non-zero map $\mathrm{L} \phi^{*} M \rightarrow \mathcal{O}_{B_{k} \mathbb{G}_{a}}$. But the functor $\mathrm{L} \phi^{*}$ sends compact objects of $\mathrm{D}_{\mathrm{qc}}(X)$ to compact objects of $\mathrm{D}_{\mathrm{qc}}\left(B_{k} \mathbb{G}_{a}\right)$ HR17, Ex. 3.8 \& Thm. 2.6(iii)]. By Proposition 3.1 it follows that $\mathrm{L} \phi^{*} M \simeq 0$ and we have a contradiction. Hence $\mathrm{D}_{\mathrm{qc}}(X)$ is not compactly generated.

Now assume that $X$ is of global type, is noetherian or has affine diagonal, and $\mathrm{D}(\mathrm{QCoh}(X))$ is compactly generated. It follows that there is a compact object $M \in \mathrm{D}(\mathrm{Q} \operatorname{Coh}(X))$ and a non-zero map $M \rightarrow \mathrm{R}\left(\phi_{\mathrm{Q} C o h}\right)_{*} \mathcal{O}_{B_{k} \mathbb{G}_{a}}$. By assumption, there is an étale covering $p: X^{\prime} \rightarrow X$ such that $X^{\prime}$ has affine diagonal and the resolution property. By Lemma 4.2 3, we may assume that $\phi$ factors through a map $\phi^{\prime}: B_{k} \mathbb{G}_{a} \rightarrow X^{\prime}$. Since $X$ is of global type, if it is noetherian, then it is affine-pointed. It follows immediately from Proposition 2.1 that

$$
\mathrm{R}\left(\phi_{\mathrm{QCoh}}\right)_{*} \mathcal{O}_{B_{k} \mathbb{G}_{a}} \simeq \mathrm{R}\left(p_{\mathrm{QCoh}}\right)_{*} \mathrm{R}\left(\phi_{\mathrm{QCoh}}^{\prime}\right)_{*} \mathcal{O}_{B_{k} \mathbb{G}_{a}} .
$$

Since $p$ is flat, Lemma 4.3 implies that a left adjoint $\mathrm{L} p_{\mathrm{Q} \text { Coh }}^{*}$ to $\mathrm{R}\left(p_{\mathrm{QCoh}}\right)_{*}$ exists. Moreover, $p$ is concentrated, so $\mathrm{L} p_{\mathrm{Q}}^{*} \mathrm{Coh}$ takes compacts to compacts (Corollary 2.2 4 $)$. Also, $X^{\prime}$ has affine diagonal and the resolution property, so Lemma 4.3 implies that a left adjoint $\mathrm{L}\left(\phi_{\mathrm{QCoh}}^{\prime}\right)_{*}$ to $\mathrm{R}\left(\phi_{\mathrm{QCoh}}^{\prime}\right)_{*}$ exists and we also see that $\mathrm{L}^{\prime}{ }_{\mathrm{Q}}^{*} \mathrm{Coh}$ takes compacts to compacts. Adjunction produces a non-zero morphism $\mathrm{L} \phi_{\mathrm{QCoh}}^{\prime *} \mathrm{~L} p_{\mathrm{QCoh}}^{*} M \rightarrow \mathcal{O}_{B_{k} \mathbb{G}_{a}}$ from a compact object of $\mathrm{D}\left(\mathrm{Q} \operatorname{Coh}\left(B_{k} \mathbb{G}_{a}\right)\right)$. This contradicts Proposition 3.1

To prove Theorem 1.12, we required the following Lemma.

Lemma 4.3. Let $p: X^{\prime} \rightarrow X$ be a morphism of algebraic stacks. A left adjoint to $\mathrm{R}\left(p_{\mathrm{QCoh}}\right)_{*}: \mathrm{D}\left(\mathrm{Q} \operatorname{Coh}\left(X^{\prime}\right)\right) \rightarrow \mathrm{D}(\mathrm{Q} \operatorname{Coh}(X))$ exists if

(1) $p$ is flat; or

(2) both $X^{\prime}$ and $X$ are quasi-compact with affine diagonal and either:

(a) $X$ has the resolution property; or

(b) $\mathrm{Q} C o h(X)$ has enough flats; or

(c) $\mathrm{K}(\mathrm{Q} C o h(X))$, the homotopy category of unbounded complexes in $\mathrm{QCoh}(X)$, has enough K-flats.

Proof. By deriving the adjunction between $p^{*}$ and $p_{*}$, it is sufficient to prove that $\mathrm{L} p_{\mathrm{Q} \text { Coh }}^{*}$ exists, that is, $p^{*}$ admits a left derived functor, under one of the four additional conditions. If $p$ is flat, then $p^{*}$ is exact and this is clear. If $X$ has the resolution property, then $\mathrm{Q} \operatorname{Coh}(X)$ obviously has enough flats (i.e., every quasicoherent sheaf is the quotient of a flat quasi-coherent sheaf). Also, if QCoh $(X)$ has enough flats, then $\mathrm{K}(\mathrm{Q} \operatorname{Coh}(X))$ has enough K-flats [Spa88, Thm. 3.4]. Hence, it is sufficient to prove the existence of $\mathrm{L} p_{\mathrm{QC}}^{*}$ when $\mathrm{K}(\mathrm{Q} \operatorname{Coh}(X))$ has enough K-flats. It is sufficient to prove: if $M \in \mathrm{K}(\mathrm{Q} \operatorname{Coh}(X))$ is $\mathrm{K}$-flat and acyclic, then $p^{*} M$ is acyclic. Now the acyclicity of $p^{*} M$ is local on $X^{\prime}$, so we may assume that $X^{\prime}$ is an affine scheme. Since $X$ has affine diagonal, it follows that $p$ is now affine. In particular, it is sufficient to prove that $p_{*} p^{*} M$ is acyclic. But $\left(p_{*} \mathcal{O}_{X^{\prime}}\right) \otimes_{\mathcal{O}_{X}} M \cong p_{*} p^{*} M$ and $M$ is $\mathrm{K}$-flat, so $p_{*} p^{*} M$ is acyclic.

Remark 4.4. The proof of Theorem 1.1 2 can be varied to prove the following assertion: if $X$ is a poorly stabilized algebraic stack that is quasi-compact with affine diagonal or noetherian and affine-pointed, then there exists a closed substack $i: Z \hookrightarrow X$ such that $Z$ is poorly stabilized and $\mathrm{D}(\mathrm{Q} \operatorname{Coh}(Z))$ is not compactly generated. The only obstruction to taking $i$ to be the identity morphism in this level of generality is that we do not know when a left adjoint to $\mathrm{R}\left(i_{\mathrm{QCoh}}\right)_{*}$ exists. 
Proof of Theorem 1.3. By Lemma 4.2, there exists a field of characteristic $p>0$ and a quasi-affine morphism $\phi: B_{k} \mathbb{G}_{a} \rightarrow X$. By Corollary 2.2, there exists an integer $n \geq 1$ such that if $N \in \mathrm{Q} \operatorname{Coh}\left(B_{k} \mathbb{G}_{a}\right)$, then $\mathrm{R}\left(\phi_{\mathrm{QCoh}}\right)_{*} N \in \mathrm{D}^{[0, n-1]}(\mathrm{Q} \operatorname{Coh}(X))$. By Nee11, Thm. 1.1], there exists $M \in \mathrm{Q} \operatorname{Coh}\left(B_{k} \mathbb{G}_{a}\right)$ such that the natural map in $\mathrm{D}\left(\mathrm{Q} \operatorname{Coh}\left(B_{k} \mathbb{G}_{a}\right)\right)$ :

$$
\bigoplus_{i \geq 0} M[i n] \rightarrow \prod_{i \geq 0} M[i n]
$$

is not a quasi-isomorphism - note that while Nee11, Thm. 1.1] only proves the above assertion in the case where $n=1$, a simple argument by induction on $n$ gives the claim above. Lemma 4.5 now implies that the natural map:

$$
\bigoplus_{i \geq 0} \mathrm{R}\left(\phi_{\mathrm{QCoh}}\right)_{*} M[i n] \rightarrow \prod_{i \geq 0} \mathrm{R}\left(\phi_{\mathrm{QCoh}}\right)_{*} M[i n]
$$

is not a quasi-isomorphism. Since $\mathrm{R}\left(\phi_{\mathrm{Q} C o h}\right)_{*} M \in \mathrm{D}^{[0, n-1]}(\mathrm{Q} \operatorname{Coh}(X))$, it follows that $\mathrm{D}(\mathrm{Q} \operatorname{Coh}(X))$ is not left-complete. By Remark 2.7, we have established that $\Psi_{X}$ is not fully faithful. To prove that $\Psi_{X}$ is not full, we will have to argue further.

Let $L=\mathrm{R}\left(\phi_{\mathrm{QCoh}}\right)_{*} M, S=\oplus_{i \geq 0} L[i n]$, and $P=\prod_{i>0} L[i n]$. Also, $\Phi_{X} \Psi_{X}(S) \simeq P$ (Lemma 2.6 2) ). If $\Psi_{X}$ is full, then there exists a map $P \rightarrow S$ such that the induced map $P \rightarrow S \rightarrow \Phi_{X} \Psi_{X}(S) \simeq P$ is the identity morphism. That is, $P$ is a direct summand of $S$. Since $\prod_{i \geq 0} M[i n]$ is not bounded above [Nee11, Rem. 1.2] and $\phi$ is quasi-affine, it follows that $P$ is not bounded above. But $S$ is bounded above, so $P$ cannot be a direct summand of $S$; hence, we have a contradiction and $\Psi_{X}$ is not full.

In the proof we used the following lemma in the special case where $X$ and $Y$ have affine diagonals or are noetherian and affine-pointed. Then it is a direct consequence of [HR17, Cor. 2.8] and Corollary 2.2.

Lemma 4.5. If $\phi: X \rightarrow Y$ is a quasi-affine morphism of algebraic stacks, then $\mathrm{R}\left(\phi_{\mathrm{QCoh}}\right)_{*}: \mathrm{D}(\mathrm{Q} \operatorname{Coh}(X)) \rightarrow \mathrm{D}(\mathrm{Q} \operatorname{Coh}(Y))$ is conservative.

Proof. Since $\phi$ is quasi-affine, there is a factorization of $\phi$ as $X \stackrel{j}{\rightarrow} \bar{X} \stackrel{\bar{\phi}}{\rightarrow} Y$, where $j$ is a quasi-compact open immersion and $\bar{\phi}$ is affine. Let $I \in \mathrm{K}(\mathrm{Q} \operatorname{Coh}(X))$ be a K-injective complex. Since $j_{*}: \mathrm{Q} \operatorname{Coh}(X) \rightarrow \mathrm{Q} \operatorname{Coh}(\bar{X})$ has an exact left adjoint $j^{*}$, it follows immediately that $j_{*} I$ is $\mathrm{K}$-injective in $\mathrm{K}(\mathrm{Q} \operatorname{Coh}(\bar{X}))$. In particular since $\bar{\phi}$ is affine, it follows that $\mathrm{R}\left(\phi_{\mathrm{QCoh}}\right)_{*} \simeq \mathrm{R}\left(\bar{\phi}_{\mathrm{QCoh}}\right)_{*} \circ \mathrm{R}\left(j_{\mathrm{QCoh}}\right)_{*}$. Hence to prove the conservativity of $\mathrm{R}\left(\phi_{\mathrm{Q} C o h}\right)_{*}$, we may assume that either $\phi$ is a quasi-compact open immersion or affine. The affine case is trivial. And when $\phi$ is a quasi-compact open immersion, we simply observe that $\phi^{*} \phi_{*} I \simeq I$.

\section{Appendix A. $\mathrm{D}_{\mathcal{M}}(\mathcal{A})$ IS WELl Generated}

We begin with a general lemma.

Lemma A.1. Let $\mathcal{T}$ be a well generated triangulated category and let $\mathcal{S} \subseteq \mathcal{T}$ be a localizing subcategory. The category $\mathcal{S}$ is well generated if and only if there is a set of generators in $\mathcal{S}$. That is: $\mathcal{S}$ is well generated if and only if there is a set of objects $S \subseteq \mathcal{S}$ such that any nonzero object $y \in \mathcal{S}$ admits a nonzero map $s \rightarrow y$, with $s \in S$.

Proof. If $\mathcal{S}$ is well generated then it has a set of generators $S$ satisfying a bunch of properties, one of which is that $S$ detects nonzero objects - see the definitions in [Nee01b, pp. 273-274]. What needs proof is the reverse implication.

Suppose therefore that $\mathcal{S}$ contains a set of objects $S$ as in the Lemma, that is every nonzero object $y \in \mathcal{S}$ admits a nonzero map $s \rightarrow y$ with $s \in S$. By Nee01b, Prop. 8.4.2] the set $S$ is contained in $\mathcal{T}^{\alpha}$ for some regular cardinal $\alpha$. If $\mathcal{L}=\operatorname{Loc}(S)$ 
is the localizing subcategory generated by $S$ then [Nee01b, Thm. 4.4.9] informs us that $\mathcal{L}$ is well generated. Since $S \subseteq \mathcal{S}$ and $\mathcal{S}$ is localizing it follows that $\mathcal{L} \subseteq \mathcal{S}$.

We know that $\mathcal{L}$ is well generated; to finish the proof it suffices to show that the inclusion $\mathcal{L} \subseteq \mathcal{S}$ is an equality. In any case the inclusion is a coproduct-preserving functor from the well generated category $\mathcal{L}$ and must have a right adjoint. For every object $y \in \mathcal{S}$, Nee01b, Prop. 9.1.8] tells us that there is a triangle in $\mathcal{S}$

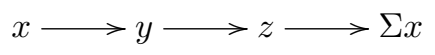

with $x \in \mathcal{L}$ and $z \in \mathcal{L}^{\perp}$. Since $z \in \mathcal{L}^{\perp} \subseteq S^{\perp}$ we have that the morphisms $s \rightarrow z$, with $s \in S$, all vanish. By the hypothesis of the Lemma it follows that $z=0$, and hence $y \cong x$ belongs to $\mathcal{L}$.

Remark A.2. We specialize Lemma A.1 to the situation where $\mathcal{T}=\mathrm{D}(\mathcal{A})$ is the derived category of a Grothendieck abelian category $\mathcal{A}$; by Nee01a, Thm. 0.2] we know that $\mathcal{T}$ is well generated, and Lemma A.1 informs us that a localizing subcategory of $\mathrm{D}(\mathcal{A})$ is well generated if and only if it has a set of generators.

Let $\mathcal{A}$ be an abelian category and fix a fully faithful subcategory $\mathcal{C} \subseteq \mathcal{A}$. Following [Stacks, Tag 02MO] we say that

(1) $\mathcal{C}$ is a Serre subcategory if it is non-empty and if $C_{1} \rightarrow A \rightarrow C_{2}$ is an exact sequence in $\mathcal{A}$ with $C_{1}, C_{2} \in \mathcal{C}$, then $A \in \mathcal{C}$;

(2) $\mathcal{C}$ is a weak Serre subcategory if it is non-empty and if

$$
C_{1} \longrightarrow C_{2} \longrightarrow A \longrightarrow C_{3} \longrightarrow C_{4},
$$

is an exact sequence in $\mathcal{A}$, where the $C_{i} \in \mathcal{C}$ and $A \in \mathcal{A}$, then $A \in \mathcal{C}$.

Clearly, Serre subcategories are weak Serre subcategories. Also, weak Serre subcategories are automatically abelian and the inclusion $\mathcal{C} \subseteq \mathcal{A}$ is exact [Stacks, Tags $02 \mathrm{MP} \& 0754$. Moreover, the subcategory $\mathrm{D}_{\mathcal{C}}(\mathcal{A})$ of $\mathrm{D}(\mathcal{A})$, consisting of complexes in $\mathcal{A}$ with cohomology in $\mathcal{C}$, is triangulated Stacks, Tag 06UQ.

The main result of this appendix is

Theorem A.3. Let $\mathcal{A}$ be a Grothendieck abelian category and let $\mathcal{M} \subseteq \mathcal{A}$ be a weak Serre subcategory closed under coproducts. If $\mathcal{M}$ is Grothendieck abelian, then $\mathrm{D}_{\mathcal{M}}(\mathcal{A})$ is well generated.

The example we have in mind is where $X$ is an algebraic stack, $\mathcal{A}$ is the category of lisse-étale sheaves of $\mathcal{O}_{X}$-modules, and $\mathcal{M}$ is the subcategory of quasi-coherent sheaves.

Remark A.4. Note that in the $\infty$-category of $\infty$-categories, we have the following homotopy cartesian diagram:

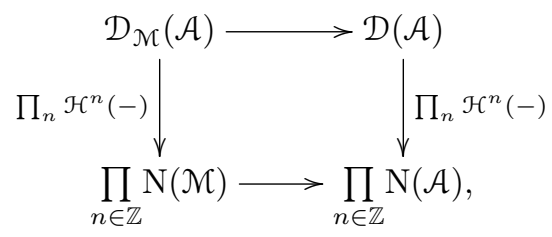

where $\mathcal{D}(\mathcal{A})$ denotes the derived $\infty$-category of $\mathcal{A}\left[\mathrm{HA}\right.$, Defn. 1.3.5.8] and $\mathcal{D}_{\mathcal{M}}(\mathcal{A})$ denotes the $\infty$-subcategory with homology in $\mathcal{M}$. This is immediate because the replete full subcategory $\mathcal{M} \subseteq \mathcal{A}$ gives rise to a categorical fibration $\mathrm{N}(\mathcal{M}) \subseteq \mathrm{N}(\mathcal{A})$ of $\infty$-categories. In particular, $\mathcal{D}_{\mathcal{M}}(\mathcal{A})$ is a presentable and stable $\infty$-category (combine [HTT, Prop. 5.5.3.12] with [HA, Prop. 1.3.5.21]). Since the homotopy category of $\mathcal{D}_{\mathcal{M}}(\mathcal{A})$ is $\mathrm{D}_{\mathcal{M}}(\mathcal{A})$, the derived category we are interested in is wellgenerated [HA, Cor. 1.4.4.2]. 
If $\lambda$ is a cardinal and $\mathcal{B}$ is a cocomplete category, then we let $\mathcal{B}^{\lambda}$ denote the subcategory of $\lambda$-presentable objects. If $\mathcal{B}$ is locally presentable, then $\mathcal{B}^{\lambda}$ is always a set.

It is clear that $\mathrm{D}_{\mathcal{M}}(\mathcal{A})$ is a localizing subcategory of the well generated triangulated category $\mathrm{D}(\mathcal{A})$; Remark A.2 tells us that to prove Theorem A.3 it suffices to exhibit a set $S$ of generators for $\mathrm{D}_{\mathcal{M}}(\mathcal{A})$. The idea is simple enough: we will find a cardinal $\lambda$ such that $S=\mathrm{D}_{\mathcal{M}^{\lambda}}^{-}\left(\mathcal{A}^{\lambda}\right) \subseteq \mathrm{D}_{\mathcal{M}}(\mathcal{A})$, which is obviously essentially small, suffices. Thus, the problem becomes to better understand the category of $\lambda$-presentable objects in $\mathcal{A}$. The results below are easy to extract from [Nee14], but for the reader's convenience we give a self-contained treatment.

Remark A.5. Let $\mathcal{A}$ be a Grothendieck abelian category. By the Gabriel-Popescu theorem, there exists a ring $R$ and a pair of adjoint additive functors

$$
F: \operatorname{Mod}(R) \longrightarrow \mathcal{A}: G
$$

such that $F$ is exact and $F G \simeq$ id. Let $\mu$ be an infinite cardinal $\geq$ to the cardinality of $R$.

Lemma A.6. With notation as in Remark $A .5$, let $\lambda>\mu$ be a regular cardinal. Then the $\lambda$-presentable objects of $\mathcal{A}$ are precisely the objects of $\mathcal{A}$ isomorphic to some $F N$, where $N$ is an $R$-module of cardinality $<\lambda$.

Proof. Let us first prove that, if $N$ is an $R$-module of cardinality $<\lambda$, then $F N$ is $\lambda$-presentable. Suppose $\left\{x_{i}, i \in I\right\}$ is a $\lambda$-filtered system in $\mathcal{A}$, and suppose that in the category $\mathcal{A}$ we are given a map $\phi: F N \longrightarrow \operatorname{colim} x_{i}$. We need to show that $\phi$ factors through some $x_{i}$. In the category of $R$-modules, there is a natural map

$$
\operatorname{colim} G x_{i} \stackrel{\rho}{\longrightarrow} G\left[\operatorname{colim} x_{i}\right] \text {. }
$$

Since $F$ respects colimits and $F G \simeq$ id, the map $F \rho$ is an isomorphism. As $F$ is exact it must annihilate the kernel and cokernel of $\rho$. Form the pullback square

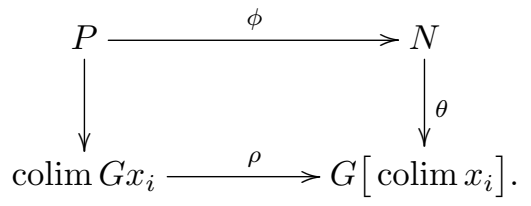

The image of $\phi$ is a submodule of $N$, hence has cardinality $<\lambda$. If we lift every element of Image $(\phi)$ arbitrarily to an element of $P$, the lifts generate a submodule $M \subseteq P$ of cardinality $<\lambda$. The kernel (respectively cokernel) of the map $M \rightarrow N$ is a submodule of $\operatorname{Kernel}(\rho)$ (respectively Coker $(\rho)$ ), and hence both are annihilated by $F$. Summarizing: we have produced a morphism $M \rightarrow N$ of $R$-modules, with $M$ of cardinality $<\lambda$ and $F M \rightarrow F N$ an isomorphism in $\mathcal{A}$, and such that the composite $M \rightarrow N \longrightarrow G\left[\operatorname{colim} x_{i}\right]$ factors through colim $G x_{i}$. But $\left\{G x_{i}, i \in I\right\}$ is a $\lambda$-filtered system in $\operatorname{Mod}(R)$ and $M$ is of cardinality $<\lambda$, and hence the map factors as $M \rightarrow G x_{i}$ for some $i \in I$.

It remains to prove the converse: suppose $a \in \mathcal{A}$ is a $\lambda$-presentable object. Then $G a$ is an $R$-module, hence it is the $\lambda$-filtered colimit of all its submodules $N_{i}$ of cardinality $<\lambda$. But then the identity map $a \rightarrow a$ is a map from the $\lambda$-presentable object $a$ to the $\lambda$-filtered colimit of the $F N_{i}$, and therefore factors through some $F N_{i}$. Thus $a$ is a direct summand of an object $F N_{i}$ where the cardinality of $N_{i}$ is $<\lambda$. On the other hand the map $N_{i} \rightarrow G a$ is injective, hence so is $F N_{i} \rightarrow F G a=a$. Thus $a \cong F N_{i}$.

Lemma A.7. Let $\mathcal{A}$ be a Grothendieck abelian category. There is an infinite cardinal $\nu$ with the following properties: if $\lambda \geq \nu$ is a regular cardinal, then 
(1) $\mathcal{A}^{\lambda}$ is a Serre subcategory of $\mathcal{A}$;

(2) every object of $\mathcal{A}$ is a $\lambda$-filtered colimit of subobjects belonging to $\mathcal{A}^{\lambda}$;

(3) an object belongs to $\mathcal{A}^{\lambda}$ if and only if it is the quotient of a coproduct of $<\lambda$ objects of $\mathcal{A}^{\nu}$; and

(4) any pair of morphisms $x \rightarrow y \leftarrow n$ in $\mathcal{A}$, where $x \rightarrow y$ is epi and $n \in \mathcal{A}^{\lambda}$, may be completed to a commutative square

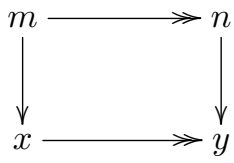

with $m \in \mathcal{A}^{\lambda}$ and $m \rightarrow n$ epi. Moreover, if $n \rightarrow y$ is mono, then $m \rightarrow x$ can be chosen to be mono.

Proof. Let $\nu=\mu+1$ be the successor of the infinite cardinal $\mu$ of Remark A.5. By Lemma A.6 the objects of $\mathcal{A}^{\lambda}$ are precisely the ones isomorphic to $F M$ where $M$ is of cardinality $<\lambda$.

For (1), it is readily verified that a subobject (resp. a quotient) of $F M$ can be expressed as $F N$ where $N$ is a submodule (resp. a quotient) of $M$. This shows that $\mathcal{A}^{\lambda}$ is closed under taking subobjects and quotients; we will later see that it is also closed under extensions.

For (2), if $a$ is an object of $\mathcal{A}$ then $G a$ is the $\lambda$-filtered colimit of all the submodules $M_{i} \subseteq G a$ of cardinality $<\lambda$, and $a \cong F G a$ is the $\lambda$-filtered colimit of $F M_{i} \in \mathcal{A}^{\lambda}$.

For (3), observe that any coproduct of $<\lambda$ objects in $\mathcal{A}^{\lambda}$ belongs to $\mathcal{A}^{\lambda}$, and if $M$ is a module of cardinality $<\lambda$ then $M$ is a quotient of the free module on all its elements, which is a coproduct of $<\lambda$ copies of $R$. Thus $F M$ is the quotient of a coproduct of $<\lambda$ copies of $F R \in \mathcal{A}^{\nu}$.

For (4), let $x \rightarrow y \leftarrow n$ be a pair of morphisms in $\mathcal{A}$, with $x \rightarrow y$ epi and $n \in \mathcal{A}^{\lambda}$. Let $\tilde{m}$ be the pullback of $n \rightarrow y$ along $x \rightarrow y$. It is sufficient to find a subobject $m$ of $\tilde{m}$ belonging to $\mathcal{A}^{\lambda}$ such that $m \rightarrow \tilde{m} \rightarrow n$ is epi. By (2), we may express $\tilde{m}=$ colim $m_{i}$ as a $\lambda$-filtered colimit of subobjects belonging $\mathcal{A}^{\lambda}$. If $n_{i} \subseteq n$ is the image of $m_{i}$ in $n$, then (1) implies that $n_{i} \in \mathcal{A}^{\lambda}$. Since $n \in \mathcal{A}^{\lambda}$, there is an $i$ such that $n_{i}=n$. Taking $m=m_{i}$ does the job. By construction, if $n \rightarrow y$ is mono, then $m \rightarrow x$ is mono.

Finally, to show that $\mathcal{A}^{\lambda}$ is closed under extensions, we note that if $0 \rightarrow k \rightarrow$ $x \rightarrow n \rightarrow 0$ is an exact sequence with $k, n \in \mathcal{A}^{\lambda}$, then (4) implies that there is a subobject $m$ of $x$ such that $m \in \mathcal{A}^{\lambda}$ and $m \rightarrow n$ is epi. It follows that $k \oplus m \rightarrow x$ is epi and consequently, $x \in \mathcal{A}^{\lambda}$, as required.

Proof of Theorem A.3. Because $\mathcal{M}$ and $\mathcal{A}$ are both Grothendieck abelian categories we may choose regular cardinals $\nu$ for $\mathcal{M}$ and $\nu^{\prime}$ for $\mathcal{A}$ as in Lemma A.7. The category $\mathcal{M}^{\nu}$ is an essentially small subcategory of $\mathcal{A}$, hence must be contained in $\mathcal{A}^{\beta}$ for some regular cardinal $\beta$. Let $\lambda$ be a regular cardinal $>\max \left(\beta, \nu, \nu^{\prime}\right)$. By construction $\mathcal{M}^{\nu} \subseteq \mathcal{A}^{\lambda}$, and by Lemma A.7 every object in $\mathcal{M}^{\lambda}$ is the quotient of a coproduct of $<\lambda$ objects in $\mathcal{M}^{\nu}$. Hence $\mathcal{M}^{\lambda} \subseteq \mathcal{A}^{\lambda}$. But since every object in $\mathcal{M} \cap \mathcal{A}^{\lambda}$ is $\lambda$-presentable in $\mathcal{A}$ it must be $\lambda$-presentable in the smaller $\mathcal{M}$, and we conclude that $\mathcal{M} \cap \mathcal{A}^{\lambda}=\mathcal{M}^{\lambda}$.

We have now made our choice of $\lambda$ and we let $\mathcal{B}=\mathcal{A}^{\lambda}$. By Remark A.2 it suffices to show that, given any non-zero object $Z \in \mathrm{D}_{\mathcal{M}}(\mathcal{A})$, there is an object $N \in \mathrm{D}_{\mathcal{B} \cap \mathcal{M}}^{-}(\mathcal{B})$ and a non-zero map $N \rightarrow Z$. If $Z$ is the chain complex

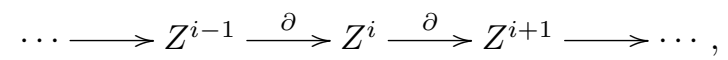


we let $Y^{i} \subseteq Z^{i}$ be the cycles, in other words the kernel of $\partial: Z^{i} \rightarrow Z^{i+1}$, and $X^{i} \subseteq Y^{i}$ be the boundaries, that is the image of $\partial: Z^{i-1} \rightarrow Z^{i}$. We are assuming that $Z \in \mathrm{D}_{\mathcal{M}}(\mathcal{A})$ is non-zero, meaning its cohomology is not all zero; without loss of generality we may assume $H^{0}(Z) \neq 0$. Thus $Y^{0} / X^{0}$ is a non-zero object of $\mathcal{M}$.

By Lemma A.7, applied to $\mathcal{B} \cap \mathcal{M}=\mathcal{M}^{\lambda} \subseteq \mathcal{M}$, the object $Y^{0} / X^{0} \in \mathcal{M}$ is a $\lambda$-filtered colimit of its subobjects belonging to $\mathcal{B} \cap \mathcal{M}$; since $Y^{0} / X^{0} \neq 0$ we may choose a subobject $M \subseteq Y^{0} / X^{0}$, with $M \in \mathcal{B} \cap \mathcal{M}$ and $M \neq 0$. By Lemma A.7. applied to the pair of maps $Y^{0} \rightarrow Y^{0} / X^{0} \leftarrow M$ in $\mathcal{A}$, we may complete to a commutative square

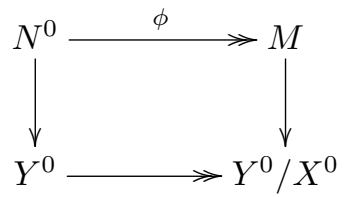

with $N^{0} \in \mathcal{B}$. Since $Y^{0}$ is the kernel of $Z^{0} \rightarrow Z^{1}$ this gives us a commutative square

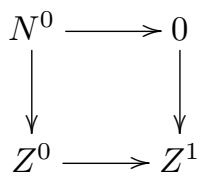

such that the image of the map $N^{0} \rightarrow Y^{0} / X^{0}=H^{0}(Z)$ is non-zero and belongs to $\mathcal{B} \cap \mathcal{M}$.

We propose to inductively extend this to the left. We will define a commutative diagram

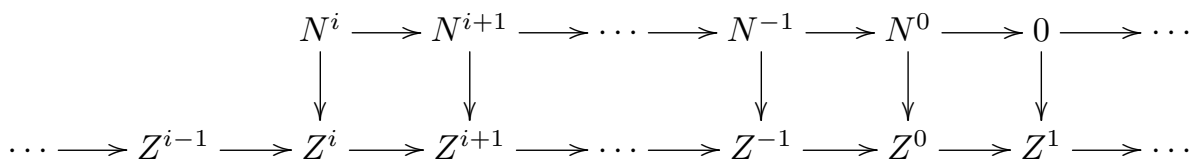

where

(1) The subobjects $N^{j} \subseteq Z^{j}$ belong to $\mathcal{B}$.

(2) For $j>i$ the cohomology of $N^{j-1} \rightarrow N^{j} \rightarrow N^{j+1}$ belongs to $\mathcal{B} \cap \mathcal{M}$.

(3) Let $K^{i}$ be the kernel of the map $N^{i} \rightarrow N^{i+1}$. Then the image of the natural map $K^{i} \rightarrow H^{i}(Z)$ belongs to $\mathcal{B} \cap \mathcal{M}$.

Since we have constructed $N^{0}$ we only need to prove the inductive step. Let us therefore suppose we have constructed the diagram as far as $i$; we need to extend it to $i-1$. We first form the pullback square

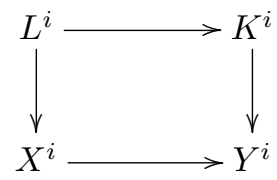

Since $X^{i} \rightarrow Y^{i}$ and $K^{i} \rightarrow Y^{i}$ are monomorphisms so are $L^{i} \rightarrow K^{i}$ and $L^{i} \rightarrow X^{i}$. Since $N^{i}$ belongs to $\mathcal{B}$ so do its subobjects $L^{i} \subseteq K^{i}$. The cokernel of $L^{i} \rightarrow K^{i}$ is the image of $K^{i} \rightarrow Y^{i} / X^{i}=H^{i}(Z)$, and belongs to $\mathcal{B} \cap \mathcal{M}$ by (3). Next we apply Lemma A.7 to the pair of maps $Z^{i-1} / X^{i-1} \rightarrow X^{i} \leftarrow L^{i}$ in $\mathcal{A}$, completing to a commutative square

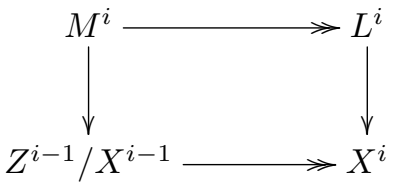


with $M^{i} \in \mathcal{B}$. Form the pullback

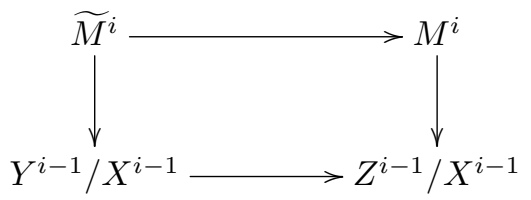

Since $Y^{i-1} / X^{i-1} \rightarrow Z^{i-1} / X^{i-1}$ is injective so is $\widetilde{M}^{i} \rightarrow M^{i}$, making $\widetilde{M}^{i}$ a subobject of $M^{i} \in \mathcal{B}$. Hence $\widetilde{M}^{i}$ belongs to $\mathcal{B}$. But now the map $\widetilde{M}^{i} \rightarrow Y^{i-1} / X^{i-1}=$ $H^{i-1}(Z)$ is a morphism from the $\lambda$-presentable object $\widetilde{M}^{i} \in \mathcal{B}=\mathcal{A}^{\lambda}$ to the object $H^{i-1}(Z) \in \mathcal{M}$, which by Lemma A.7 is a $\lambda$-filtered colimit of its subobjects in $\mathcal{M}^{\lambda}=\mathcal{B} \cap \mathcal{M}$. Hence the map $\widetilde{M^{i}} \rightarrow Y^{i-1} / X^{i-1}$ factors as $\widetilde{M}^{i} \rightarrow P^{i} \rightarrow Y^{i-1} / X^{i-1}$ with $P^{i} \in \mathcal{B} \cap \mathcal{M}$ a subobject of $Y^{i-1} / X^{i-1}$. Form the pushout square in $\mathcal{B}$

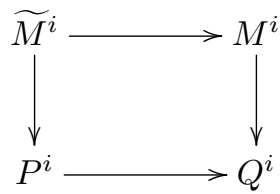

and let $Q^{i} \rightarrow Z^{i-1} / X^{i-1}$ be the natural map. We have a commutative square

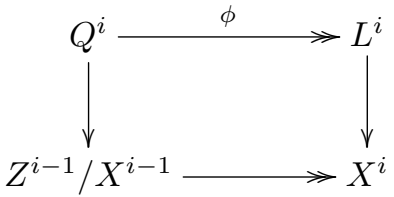

and the kernel of $\phi$ maps isomorphically to the subobject $P^{i} \subseteq H^{i-1}(Z)$, with $P^{i} \in \mathcal{B} \cap \mathcal{M}$. Finally apply Lemma A.7 to the pair of maps $Z^{i-1} \rightarrow Z^{i-1} / X^{i-1} \leftarrow Q^{i}$ to complete to a square

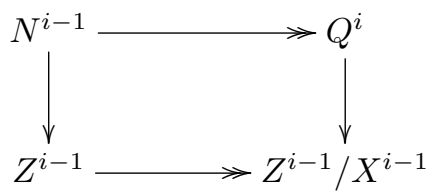

with $N^{i} \in \mathcal{B}$. We leave it to the reader to check that the diagram

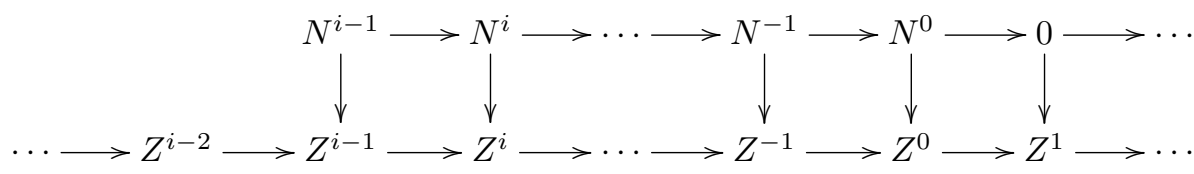

satisfies hypotheses (1), (2) and (3) of our induction.

Appendix B. $\mathrm{D}_{\mathrm{qc}}(X)$ IS LEFT-COMPLETE

Let $\mathcal{T}$ be a triangulated category with a $t$-structure $(\mathcal{T} \leq 0, \mathcal{T} \geq 0)$. If $\mathcal{T}$ admits countable projects, then we say that the $t$-structure is left-complete if the map

$$
M \rightarrow \underset{n}{\operatorname{holim}} \tau^{\geq-n} M
$$

is an isomorphism for every $M \in \mathcal{T}$. A thorough discussion of $t$-structures using the language of stable $\infty$-categories is available in [HA, $\S 1.2 .1]$.

If $\mathcal{A}$ is a Grothendieck abelian category, then the standard $t$-structure on $\mathrm{D}(\mathcal{A})$ is $\left(\mathrm{D} \leq 0(\mathcal{A}), \mathrm{D}^{\geq 0}(\mathcal{A})\right)$. If $\mathcal{M} \subseteq \mathcal{A}$ is a Grothendieck abelian weak Serre subcategory, 
then the standard $t$-structure on $\mathrm{D}_{\mathcal{M}}(\mathcal{A})$ is the one induced by restriction from $\mathrm{D}(\mathcal{A})$. If no $t$-structure on $\mathrm{D}_{\mathcal{M}}(\mathcal{A})$ is specified, then we will always mean the standard one.

In this section we prove the following Theorem.

Theorem B.1. If $X$ is an algebraic stack, then $\mathrm{D}_{\mathrm{qc}}(X)$ is well generated. In particular, it admits small products. Moreover, $\mathrm{D}_{\mathrm{qc}}(X)$ is left-complete.

Proof. The subcategory $\mathrm{Q} \operatorname{Coh}(X) \subseteq \operatorname{Mod}\left(X_{\text {lis-ét }}\right)$ is weak Serre and the inclusion is coproduct preserving. Since $\mathrm{Q} \operatorname{Coh}(X)$ and $\operatorname{Mod}\left(X_{\text {lis-ét }}\right)$ are Grothendieck abelian categories [Stacks, Tags 07A5 \& 0781], it follows that $\mathrm{D}_{\mathrm{qc}}(X)$ is well generated (Theorem A.3. In particular, $\mathrm{D}_{\mathrm{qc}}(X)$ admits small products [Nee01b, Cor. 1.18].

It remains to prove that $\mathrm{D}_{\mathrm{qc}}(X)$ is left-complete, which we accomplish by extracting from [Stacks, Tag 08U3 a useful special case (which was communicated to us by Bhatt Bha12). So the inclusion $\omega: \mathrm{D}_{\mathrm{qc}}(X) \rightarrow \mathrm{D}(X)$ is exact and coproduct preserving; thus, the functor $\omega$ admits a right adjoint $\lambda$ [Nee01b, Prop. 1.20]. Because the functor $\omega$ is fully faithful, the adjunction id $\Rightarrow \lambda \circ \omega$ is an isomorphism of functors.

Note that because $\lambda$ is a right adjoint, it preserves products and so homotopy limits. In particular, it remains to prove that if $K \in \mathrm{D}_{\mathrm{qc}}(X)$, then a naturally induced map:

$$
c: \omega(K) \rightarrow \underset{n}{\operatorname{holim}} \tau^{\geq-n} \omega(K)
$$

is a quasi-isomorphism in $\mathrm{D}(X)$ (where we also take the homotopy limit in $\mathrm{D}(X)$ ). Indeed, this follows from the observation that $\tau^{\geq-n} \omega(K) \simeq \omega\left(\tau^{\geq-n} K\right)$ for all integers $n$ and $K \rightarrow \lambda \circ \omega(K)$ is an isomorphism.

To see that $c$ is a quasi-isomorphism in $\mathrm{D}(X)$, it is sufficient to prove that $\operatorname{RHom}_{\mathcal{O}_{X}}\left(p_{!} \mathcal{O}_{U}, c\right)$ is a quasi-isomorphism for every smooth morphism $p: U \rightarrow X$, where $U$ is an affine scheme. Observe that

$$
\operatorname{RHom}_{\mathcal{O}_{X}}\left(p_{!} \mathcal{O}_{U}, \tau^{\geq-n} K\right) \simeq \operatorname{RHom}_{\mathcal{O}_{U}}\left(\mathcal{O}_{U}, \tau^{\geq-n} p^{-1} K\right) \simeq \tau^{\geq-n} K(U),
$$

with the final quasi-isomorphism because $U$ is affine and $K$ has quasi-coherent cohomology Stacks, Tags 01XB \& 0756. But RHom $\mathcal{O}_{X}\left(p_{!} \mathcal{O}_{U},-\right)$ commutes with homotopy limits, so it is sufficient to prove that we have the following quasi-isomorphism of abelian groups:

$$
K(U) \rightarrow \underset{n}{\operatorname{holim}} \tau^{\geq-n} K(U),
$$

which is well-known, because the products in the category of abelian groups are exact. For details, see for example [Stacks, Tag 07KC].

\section{Appendix C. The Bounded Below Derived CATEgory}

In this section, we prove an analog of [Har66, Cor. II.7.19] for noetherian algebraic stacks that are affine-pointed, cf. [Lie04, Rem. 2.2.4.7]. Essentially for free, we will also establish Lurie's result Lur04, Thm. 3.8].

Theorem C.1. Let $X$ be an algebraic stack. If $X$ is either quasi-compact with affine diagonal or noetherian and affine-pointed, then the natural functor

$$
\Psi_{X}^{+}: \mathrm{D}^{+}(\mathrm{Q} \operatorname{Coh}(X)) \rightarrow \mathrm{D}_{\mathrm{qc}}^{+}(X)
$$

is an equivalence.

The conditions on $X$ are essentially sharp: $\Psi_{X}^{b}$ can fail to be fully faithful if:

(1) $X$ is a non-noetherian quasi-compact and quasi-separated scheme with nonaffine diagonal [SGA6, Exp. II, App. I].

(2) $X$ is noetherian with non-affine stabilizers, e.g., if $X$ is the classifying stack of an elliptic curve. 
For noetherian algebraic spaces, a version of Theorem C.1 for the unbounded derived category was proved in [Stacks, Tag 09TN] and we will closely follow this approach. The following two lemmas do most of the work.

Lemma C.2 (cf. [Stacks, Tag 09TJ]). Let X be a quasi-compact and quasi-separated algebraic stack and let $I$ be an injective object of $\mathrm{Q} C o h(X)$.

(1) Then $I$ is a direct summand of $p_{*} J$, where $p$ : Spec $A \rightarrow X$ is smooth and surjective and $J$ is an injective A-module.

(2) If $X$ is noetherian, then $I$ is a direct summand of a filtered colimit $\operatorname{colim}_{i} F_{i}$ of quasi-coherent sheaves of the form $F_{i}=\gamma_{i, *} G_{i}$, where $\gamma_{i}: Z_{i} \rightarrow X$ is a morphism from an artinian scheme $Z_{i}$ and $G_{i} \in \operatorname{Coh}\left(Z_{i}\right)$.

Proof. Let $p: U \rightarrow X$ be a smooth and surjective morphism, where $U=\operatorname{Spec} A$ is an affine scheme. Let $I$ be an injective object of $\mathrm{QCoh}(X)$. Choose an injective object $J$ of QCoh $(U)$ and an injection $p^{*} I \subseteq J$. By adjunction, we have an inclusion $I \subseteq p_{*} J$. Since $p^{*}$ is exact, $p_{*} J$ is injective in $\mathrm{Q} \operatorname{Coh}(X)$ and $I$ is a direct summand of $p_{*} J$. This proves (1). For (2): we may now reduce to the case where $X=U$. The result is now well-known (e.g., [Stacks, Tag 09TI).

Lemma C.3 (cf. Stacks, Tag 09TL]). Let $X$ be an algebraic stack and let $I$ be an injective object of $\mathrm{Q} C \mathrm{Ch}(X)$. If $X$ is quasi-compact with affine diagonal (resp. noetherian and affine-pointed), then

(1) $H^{q}\left(U_{\text {lis-ét }}, I\right)=0$ for every $q>0$ and smooth morphism $u: U \rightarrow X$ that is affine (resp. has affine fibers);

(2) for any morphism $f: X \rightarrow Y$ of algebraic stacks, where $Y$ has affine diagonal (resp. $Y$ is affine-pointed) we have $\mathrm{R}^{q}\left(f_{\text {lis-ét }}\right)_{*} I=0$ for $q>0$.

Proof. Let $W$ be an affine (resp. artinian) scheme and let $M \in \mathrm{Q} C o h(W)$ be injective (resp. $M \in \operatorname{Coh}(W)$ ). Let $w: W \rightarrow X$ be a smooth and surjective morphism (resp. a morphism). By Lemma C.2 it is sufficient to prove the result for $I=w_{*} M$. Since $X$ has affine diagonal (resp. $X$ is affine-pointed), $w$ is affine. In particular, the natural map $\left(w_{*} M\right)[0] \rightarrow \mathrm{R}\left(w_{\text {lis-ét }}\right)_{*} M$ is a quasi-isomorphism.

We now prove (1). Let $u_{W}: W_{U} \rightarrow W$ be the pull back of $u$ along $w$ and let $w_{U}: W_{U} \rightarrow U$ be the pull back of $w$ along $u$. In both cases, $u_{W}$ is smooth and affine and $w_{U}$ is affine; in particular, $W_{U}$ is an affine scheme. Since $u$ is smooth,

$$
\begin{aligned}
\mathrm{R} \Gamma\left(U_{\text {lis-ét }}, I\right) & \simeq \mathrm{R} \Gamma\left(U_{\text {lis-ét }}, u^{*} \mathrm{R}\left(w_{\text {lis-ét }}\right)_{*} M\right) \simeq \mathrm{R} \Gamma\left(U_{\text {lis-ét }}, \mathrm{R}\left(\left(w_{U}\right)_{\text {lis-ét }}\right)_{*}\left(u_{W}^{*} M\right)\right) \\
& \simeq \mathrm{R} \Gamma\left(\left(W_{U}\right)_{\text {lis-ét }}, M\right) .
\end{aligned}
$$

The result now follows from the affine case (e.g., [EGA, III.1.3.1]).

For (2): let $v: V \rightarrow Y$ be a smooth morphism, where $V$ is an affine scheme. Since $Y$ has affine diagonal (resp. is affine-pointed), $v$ is affine (resp. has affine fibers). By (1), $H^{q}\left(\left(V \times_{Y} X\right)_{\text {lis-ét }}, I\right)=0$. But $\mathrm{R}^{q}\left(f_{\text {lis-ét }}\right)_{*} I$ is the sheafification of the presheaf $V \mapsto H^{q}\left(\left(V \times_{Y} X\right)_{\text {lis-ét }}, I\right)$; the result follows.

Proof of Theorem C.1. We first establish that $\Psi_{X}^{+}$is fully faithful: given $F, G \in$ $\mathrm{D}^{+}(\mathrm{Q} \operatorname{Coh}(X))$ we wish to prove that the natural map

$$
\operatorname{Hom}_{\mathrm{D}(\mathrm{Q} C o h(X))}(F, G) \rightarrow \operatorname{Hom}_{\mathrm{D}(X)}(F, G)
$$

is an isomorphism. A standard way-out argument shows that it is sufficient to prove that the natural map

$$
\operatorname{Ext}_{\mathrm{Q} \operatorname{Coh}(X)}^{q}(N, M) \rightarrow \operatorname{Ext}_{\mathcal{O}_{X}}^{q}(N, M)
$$

is an isomorphism for every $q \in \mathbb{Z}$ and $M, N \in \mathrm{Q} \operatorname{Coh}(X)$. For $q<0$ both sides vanish and for $q=0$ we clearly have an isomorphism. For $q>0$, since every $M$ 
embeds in a quasi-coherent injective $I$, a standard $\delta$-functor argument shows that it is sufficient to prove that if $I$ is an injective object of $\mathrm{Q} \operatorname{Coh}(X)$, then

$$
\operatorname{Ext}_{\mathcal{O}_{X}}^{q}(N, I)=0
$$

for all $q>0$ and $N \in \mathrm{QCoh}(X)$. To see this we note that by Lemma C.2 1), $I$ is a direct summand of $\left(p_{\mathrm{QCoh}}\right)_{*} J$, where $p: \operatorname{Spec} A \rightarrow X$ is smooth and surjective and $J$ is an injective $A$-module. Thus, it suffices to prove the result when $I=$ $\left(p_{\mathrm{QCoh}}\right)_{*} J$. By Lemma C.3 2), the natural map $\left(\left(p_{\mathrm{QCoh}}\right)_{*} J\right)[0] \rightarrow \mathrm{R}\left(p_{\text {lis-ét }}\right)_{*} J$ is a quasi-isomorphism. Hence, there are natural isomorphisms:

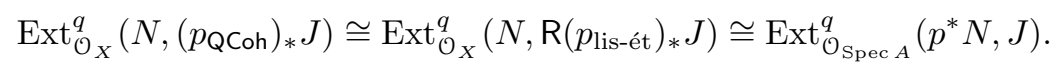

We are now reduced to the affine case, which is well-known (e.g., [BN93, Lem. 5.4]).

For the essential surjectivity, we argue as follows: by induction and using the full faithfulness, one easily sees that $\mathrm{D}^{b}(\mathrm{Q} \operatorname{Coh}(X)) \simeq \mathrm{D}_{\mathrm{qc}}^{b}(X)$. Passing to homotopy colimits, we obtain the claim.

The following observation was made by Bhatt Bha12 and a reviewer.

Remark C.4. Let $X$ be an algebraic stack that is either quasi-compact with affine diagonal or noetherian and affine-pointed. Since $\mathrm{D}_{\mathrm{qc}}(X)$ is left-complete, $\Psi_{X}$ factors uniquely through the left-completion functor $\mathrm{D}(\mathrm{Q} \operatorname{Coh}(X)) \rightarrow \widehat{\mathrm{D}}(\mathrm{Q} \operatorname{Coh}(X))$ HA, $\S 1.2 .1]$. But $\widehat{\mathrm{D}}(\mathrm{Q} \operatorname{Coh}(X))$ is also the left completion of $\mathrm{D}^{+}(\mathrm{Q} \operatorname{Coh}(X))$ and $\Psi_{X}^{+}$ is an equivalence. Hence, $\widehat{\mathrm{D}}(\mathrm{Q} \operatorname{Coh}(X)) \rightarrow \mathrm{D}_{\mathrm{qc}}(X)$ is an equivalence.

\section{REFERENCES}

[AHR15] J. Alper, J. Hall, and D. Rydh, A Luna étale slice theorem for algebraic stacks, April 2015, arXiv:1504.06467

[BB03] A. Bondal and M. Van den Bergh, Generators and representability of functors in commutative and noncommutative geometry, Mosc. Math. J. 3 (2003), no. 1, 1-36, 258.

[Bha12] B. Bhatt, private communication, Sept-Oct 2012.

[BIK11] D. J. Benson, S. B. Iyengar, and H. Krause, Stratifying modular representations of finite groups, Ann. of Math. (2) $\mathbf{1 7 4}$ (2011), no. 3, 1643-1684.

[BN93] M. Bökstedt and A. Neeman, Homotopy limits in triangulated categories, Compositio Math. 86 (1993), no. 2, 209-234.

[Bor91] A. Borel, Linear algebraic groups, second ed., Graduate Texts in Mathematics, vol. 126, Springer-Verlag, New York, 1991.

[Bri15] M. Brion, On linearization of line bundles, J. Math. Sci. Univ. Tokyo 22 (2015), no. 1, 113-147.

[BZ09] D. Ben-Zvi, Compact generation for modular representations, MathOverflow, Nov 2009, http://mathoverflow.net/q/4965

[BZFN10] D. Ben-Zvi, J. Francis, and D. Nadler, Integral transforms and Drinfeld centers in derived algebraic geometry, J. Amer. Math. Soc. 23 (2010), no. 4, 909-966.

[Con02] B. Conrad, A modern proof of Chevalley's theorem on algebraic groups, J. Ramanujan Math. Soc. 17 (2002), no. 1, 1-18.

[DG70] M. Demazure and P. Gabriel, Groupes algébriques. Tome I: Géométrie algébrique, généralités, groupes commutatifs, Masson \& Cie, Éditeur, Paris, 1970, Avec un appendice Corps de classes local par Michiel Hazewinkel.

[DG13] V. Drinfeld and D. Gaitsgory, On some finiteness questions for algebraic stacks, Geom. Funct. Anal. 23 (2013), no. 1, 149-294.

[EGA] A. Grothendieck, Éléments de géométrie algébrique, I.H.E.S. Publ. Math. 4, 8, 11, 17, 20, 24, 28, 32 (1960, 1961, 1961, 1963, 1964, 1965, 1966, 1967).

[Gro17] P. Gross, Tensor generators on schemes and stacks, Algebr. Geom. 4 (2017), no. 4, 501-522.

[HA] J. Lurie, Higher Algebra, available on homepage, May 2016.

[Hal14] J. Hall, Cohomology and base change for algebraic stacks, Math. Z. 278 (2014), no. 1-2, 401-429. 
[Har66] R. Hartshorne, Residues and duality, Lecture notes of a seminar on the work of A. Grothendieck, given at Harvard 1963/64. With an appendix by P. Deligne. Lecture Notes in Mathematics, No. 20, Springer-Verlag, Berlin, 1966.

[HR14] J. Hall and D. Rydh, Coherent Tannaka duality and algebraicity of Hom-stacks, preprint, May 2014, arXiv:1405.7680

[HR15] J. Hall and D. Rydh, Algebraic groups and compact generation of their derived categories of representations, Indiana Univ. Math. J. 64 (2015), no. 6, 1903-1923.

[HR17] J. Hall and D. Rydh, Perfect complexes on algebraic stacks, Compositio Math. 153 (2017), no. 11, 2318-2367.

[HTT] J. Lurie, Higher topos theory, Annals of Mathematics Studies, vol. 170, Princeton University Press, Princeton, NJ, 2009.

[Kri09] A. Krishna, Perfect complexes on Deligne-Mumford stacks and applications, J. KTheory 4 (2009), no. 3, 559-603.

[KS06] M. Kashiwara and P. Schapira, Categories and sheaves, Grundlehren der Mathematischen Wissenschaften [Fundamental Principles of Mathematical Sciences], vol. 332, Springer-Verlag, Berlin, 2006.

[Lie04] M. Lieblich, Moduli of twisted sheaves and generalized Azumaya algebras, ProQuest LLC, Ann Arbor, MI, 2004, Thesis (Ph.D.)-Massachusetts Institute of Technology.

[LMB] G. Laumon and L. Moret-Bailly, Champs algébriques, Ergebnisse der Mathematik und ihrer Grenzgebiete. 3. Folge., vol. 39, Springer-Verlag, Berlin, 2000.

[Lur04] J. Lurie, Tannaka duality for geometric stacks, preprint, December 2004, arXiv:math/0412266, p. 14.

[Nee96] A. Neeman, The Grothendieck duality theorem via Bousfield's techniques and Brown representability, J. Amer. Math. Soc. 9 (1996), no. 1, 205-236.

[Nee01a] A. Neeman, On the derived category of sheaves on a manifold, Doc. Math. 6 (2001), 483-488 (electronic).

[Nee01b] A. Neeman, Triangulated categories, Annals of Mathematics Studies, vol. 148, Princeton University Press, Princeton, NJ, 2001.

[Nee11] A. Neeman, Non-left-complete derived categories, Math. Res. Lett. 18 (2011), no. 5, $827-832$

[Nee14] A. Neeman, The homotopy category of injectives, Algebra Number Theory 8 (2014), no. 2, 429-456.

[Ols07] M. Olsson, Sheaves on Artin stacks, J. Reine Angew. Math. 603 (2007), 55-112.

[Ros61] M. Rosenlicht, On quotient varieties and the affine embedding of certain homogeneous spaces, Trans. Amer. Math. Soc. 101 (1961), 211-223.

[Ryd11] D. Rydh, Étale dévissage, descent and pushouts of stacks, J. Algebra 331 (2011), 194-223.

[Ryd15] D. Rydh, Noetherian approximation of algebraic spaces and stacks, J. Algebra 422 (2015), 105-147.

[Ser03] C. Serpé, Resolution of unbounded complexes in Grothendieck categories, J. Pure Appl. Algebra 177 (2003), no. 1, 103-112.

[SGA6] Théorie des intersections et théorème de Riemann-Roch, Lecture Notes in Mathematics, Vol. 225, Springer-Verlag, Berlin, 1971, Séminaire de Géométrie Algébrique du Bois-Marie 1966-1967 (SGA 6), Dirigé par P. Berthelot, A. Grothendieck et L. Illusie. Avec la collaboration de D. Ferrand, J. P. Jouanolou, O. Jussila, S. Kleiman, M. Raynaud et J. P. Serre.

[Spa88] N. Spaltenstein, Resolutions of unbounded complexes, Compositio Math. 65 (1988), no. 2, 121-154.

[Stacks] The Stacks Project Authors, Stacks Project, http://stacks.math.columbia.edu.

[Sum74] H. Sumihiro, Equivariant completion, J. Math. Kyoto Univ. 14 (1974), 1-28.

[Toë12] B. Toën, Derived Azumaya algebras and generators for twisted derived categories, Invent. Math. 189 (2012), no. 3, 581-652.

[Tot04] B. Totaro, The resolution property for schemes and stacks, J. Reine Angew. Math. 577 (2004), 1-22.

[TT90] R. W. Thomason and T. Trobaugh, Higher algebraic K-theory of schemes and of derived categories, The Grothendieck Festschrift, Vol. III, Progr. Math., vol. 88, Birkhäuser Boston, Boston, MA, 1990, pp. 247-435. 
Department of Mathematics, University of Arizona, Tucson, AZ 85721-0089, USA

E-mail address: jackhall@math.arizona.edu

Mathematical Sciences Institute, The Australian National University, Acton, ACt, 2601, Australia

E-mail address: Amnon. Neeman@anu.edu.au

KTH Royal Institute of Technology, Department of Mathematics, SE-100 44 StockHOLM, SWEDEN

E-mail address: dary@math.kth.se 